\title{
Analysis of sex-based differences in energy substrate utilization during moderate-intensity aerobic exercise
}

\author{
Antonella Cano ${ }^{1} \cdot$ Lucia Ventura $^{1} \cdot$ Gianluca Martinez $^{1} \cdot$ Lucia Cugusi $^{1} \cdot$ Marcello Caria $^{1} \cdot$ Franca Deriu $^{1,2}$ (]) \\ Andrea Manca ${ }^{1}$
}

Received: 3 February 2021 / Accepted: 24 August 2021 / Published online: 22 September 2021

(c) The Author(s) 2021

\begin{abstract}
Purpose To explore sex-based differences in energy substrate utilization during moderate-intensity aerobic exercise; to identify the underpinning candidate physiological mechanisms.

Methods Three databases were searched from inception to August 2020. Pertinent studies quantifying the utilization of substrates during moderate aerobic exercise in healthy men and reproductive-age women were considered. Studies conducted on sedentary/recreationally active and athletic populations were included and analyzed separately.

Results Thirty-five studies entered the meta-analysis (21 in sedentary/recreationally active, 14 in athletic populations). Compared to women, the respiratory exchange ratio was significantly higher both in sedentary (mean difference, MD: +0.03 ; $\mathrm{p}<0.00001)$ and athletic men (MD: $+0.02 ; p<0.0001$ ). Greater carbohydrate oxidation was observed both in sedentary (standardized MD, SMD: 0.53; $p=0.006$ ) and athletic men (SMD: $1.24 ; p<0.00001$ ). Regarding lipid substrates, sedentary men oxidized less fat than women (SMD: $-0.77 ; p=0.0002$ ), while no sex-based differences in fat oxidation were observed in athletes (SMD: $0.06 ; p=0.77$ ). Paucity of data prevented robust meta-analyses for protein sources. Sex hormones and different adrenergic activation were the most cited mechanisms to discuss sex-based differences.

Conclusions Meta-analyses confirmed that men display greater reliance on carbohydrates while women rely more on lipids to sustain moderate aerobic exercise. The latter finding was not confirmed in athletes, a novel aspect of the present study. Mechanistically driven research is needed to further dissect the physiological underpinnings of sex differences in substrate utilization during aerobic exercise, especially for proteins, which are still less investigated than other substrates.
\end{abstract}

Keywords Energy metabolism $\cdot$ Exercise physiology $\cdot$ Sex characteristics $\cdot$ Aerobic exercise

$\begin{array}{ll}\text { Abbreviations } \\ \text { CI } & \text { Confidence interval } \\ \text { FFA } & \text { Free fatty acids } \\ \text { HSL } & \text { Hormone-sensitive lipase } \\ \text { I }^{2} & \text { Inconsistency test } \\ \text { IMCL } & \text { Intramyocellular lipid } \\ \text { MD } & \text { Mean difference }\end{array}$

Communicated by Michael Lindinger .

Antonella Cano and Lucia Ventura have contributed equally to this work.

Franca Deriu

deriuf@uniss.it

1 Department of Biomedical Sciences, University of Sassari, Viale S. Pietro 43/b, 07100 Sassari, Italy

2 Unit of Endocrinology, Nutritional and Metabolic Disorders, AOU Sassari, Sassari, Italy
MeSH Medical Subject Heading

NIH National Institutes of Health

$\mathrm{O}_{2} \quad$ Oxygen

RER Respiratory exchange ratio

SD Standard deviation

SMD Standardized mean difference

$\mathrm{VO}_{2} \quad$ Volume of oxygen

\section{Introduction}

Sex-based differences are well known to exist in endurance performance where, relative to body mass and composition, females would outperform males during exercise at submaximal intensities (Hunter et al. 2014). Women, when exercising at matched intensity, display reduced muscle fatigability and metabolic advantage in comparison to men. This fact has been attributed to a higher lipolytic efficiency (Bergström 
and Hultman 1966) and to a greater relative distribution and activation of fatigue resistant slow twitch fibers (Zierath and Hawley 2004; Hunter 2014; Temesi et al. 2015; Tiller et al. 2021). Nonetheless, histological, enzymatic, and hormonal aspects must be considered for the true sex-based differences in performance and fatigability, in addition to psychological and sociological factors, which could also have a confounding effect.

\section{Sex-based differences in carbohydrate metabolism}

Sex hormones are considered key biological contributors to sex-based differences in substrate utilization. Both estrogen and progesterone alter metabolic responses, displaying opposed effects (Oosthuyse and Bosch 2010): while the former appears to impede glucose kinetics, the latter seems to potentiate it ( $\mathrm{D}^{\prime}$ Eon et al. 2002). Indeed, estrogen promotes endurance performance by hepatic glycogen sparing (Friedlander et al. 1998; Carter et al. 2001; Devries et al. 2007). High concentrations of estrogen (e.g., in the luteal phase of eumenorrheic women) can reduce reliance on muscle glycogen during moderate exercise (D'Eon et al. 2002), promoting insulin sensitivity.

One study on eumenorrheic women compared estrogen versus estrogen plus progesterone pharmacological administration and demonstrated higher total carbohydrate oxidation and muscle glycogen utilization for the latter condition (D'Eon et al. 2002). Controversially, data obtained in the luteal phase (when progesterone predominates) have shown lower muscle glycogen utilization during exercise in comparison with the follicular phase (when estrogen predominates) (Hackney 1999; Devries et al. 2006). The influence of progesterone alone on substrate utilization during endurance exercise is still uncovered.

\section{Sex-based differences in lipid metabolism}

Several investigations, conducted both in sedentary and recreationally active individuals, confirmed greater reliance on lipids in women, during aerobic exercise. Such evidence indicates that not only women oxidize significantly more lipids than men (Horton et al. 1998; McKenzie et al. 2000; Lamont et al. 2001a; Henderson et al. 2007; Tarnopolsky et al. 2007; Cheneviere et al. 2011; Dasilva et al. 2011; Isacco et al. 2012; Isacco et al. 2020), but they also use less carbohydrate and protein substrates to sustain moderate exercise (McKenzie et al. 2000; Tarnopolsky 2000; Lamont et al. 2001a, 2003; Devries 2016). Comparable findings have been obtained also in athletic, endurance-trained populations (Phillips et al. 1993; Knechtle et al. 2004; Riddell et al. 2003; Wallis et al. 2006).

During exercise, the greater mRNA expression of genes associated with free fatty acid (FFA) transport to plasma and mitochondrial membranes in females has been associated to facilitate lipid metabolism (Kiens et al. 2004; Monaco et al. 2015) and higher lipid oxidation rate (Venables et al. 2005; Chenevière et al. 2011). Whether increased lipid metabolism in women during exercise is consequent to predominant oxidation of either plasma FFA or intramyocellular lipids (IMCL) is debated (Devries 2016). Indeed, while women display significantly larger storages of IMCL than men (Roepstorff et al. 2002; Devries et al. 2007), experimental evidence is inconclusive on whether they also have greater capacity to use this substrate.

Sex-based studies examining catecholamines' effects on lipolysis, at rest, reported similar plasma concentrations and adipose tissue lipolytic sensitivity (Jensen et al. 1996; Millet et al. 1998). Different patterns of adrenergic receptor activation might be responsible for the diverse lipolysis regulation in men and women during endurance exercise (Hellström et al. 1996; Boschmann et al. 2002). Specifically, moderateintensity exercise activates both $\beta 1$ (lipolysis-activating) and $\alpha 2$ (lipolysis-inhibiting) receptors in men, whereas it activates only $\beta 1$ receptors in women (Blatchford et al. 1985; Arner et al. 1990; Davis et al. 2000).

While sex differences in carbohydrate and lipid metabolism during exercise have been extensively investigated, few and controversial data are available for protein metabolism. Some authors reported significantly larger utilization of protein sources in men than women (Phillips et al. 1993; Lamont et al. 2001a), while others failed to detect any sexbased differences (Horton et al. 1998).

\section{Controversies and potential weaknesses in the existing literature}

Several controversial findings can be traced in the available sex-comparative literature regarding the type of substrate used to sustain submaximal endurance exercise. For instance, Ruby and colleagues (2002) did not detect sexbased differences in total fat oxidation but, after data correction for body mass, fat oxidation rates were higher in men than women (Ruby et al. 2002). A highly controlled study reported greater adipose tissue triglyceride lipolysis and larger plasma FFA availability and oxidation in women than men, who were matched for percent body fat and aerobic fitness. However, the same study showed a similar total fat oxidation due to a reciprocal decrease in the oxidation rate of non-plasma-derived FFA in women (Mittendorfer et al. 2002). In line with these observations, previous studies conducted in untrained men and women with similar aerobic fitness and body fat found minimal or no difference in lipid oxidation rates (Costill et al. 1979; Powers et al. 1980; Keim et al. 1996; Horowitz and Klein 2000). Overall, body composition seems to play a role in the pattern of substrate oxidation during exercise, as the basal larger percent body fat 
in women would prompt a higher regional lipolysis (Davis et al. 2000; Cheneviere et al. 2011). Poor control of this parameter may be responsible for magnifying the sex-based differences in lipid oxidation rates generally reported.

Inconsistencies among the findings may be attributed also to poor control of training and nutritional status, to diverse methods employed to evaluate the metabolic rates, and different populations studied. Moreover, superficial characterization and consideration of the menstrual cycle phases, hormonal profile, and exogenous manipulation might lead to heterogenous female population.

The underpowered sample size of the studies threatens the validity of the findings, since results are subject to selection, information, and confounding biases, which are often poorly controlled in observational research (Grimes and Schultz 2002;; Simunovic et al. 2009). The precision and accuracy of estimates reported in individual studies can be significantly enhanced by grouping individual works and pooling their data via meta-analytic approaches, provided that the inherent heterogeneity across studies is controlled.

Despite the considerable number of reports on sex-based differences in energy substrate utilization during moderateintensity aerobic exercise, there are no synthesis works, of which we are aware, that have quantitatively examined pooled data from the pertinent literature. Additionally, such body of knowledge has not been scrutinized yet in terms of its methodological quality and the risk for biases potentially threatening this literature.

Based on the above background and rationale, we performed a meta-analytic aggregation of data from sexcomparative studies to: (1) verify the extent of sex-based differences in carbohydrate, lipid, and protein metabolism during moderate-intensity aerobic exercise; (2) qualitatively appraise, code, and count the physiological mechanisms underpinning differences in substrate utilization between men and women; (3) further explore whether sexbased responses to exercise and putative mechanisms differ depending on the training status.

\section{Methods}

The Preferred Reporting Items for Systematic Reviews and Meta-Analyses (PRISMA) guidelines and flowchart diagram were used as a reporting structure for this meta-analysis (Liberati et al. 2009).

\section{Selection of studies}

The following databases were searched to retrieve pertinent articles: PubMed (including Medline), Scopus, and Web of Science. The search combined keywords, Medical Subject Headings (MeSH) and matching synonyms relevant to the topic (metabolism OR lipids metabolism OR carbohydrates metabolism OR glycogen metabolism OR glucose metabolism OR energy metabolism OR energetic metabolism OR protein metabolism) AND Oxygen Consumption/physiology [MeSH] AND Physical Endurance/physiology [MeSH] AND (male AND female) AND (gender OR sex). Only case-control, cross-sectional, and pre-post studies carried out in healthy adults (18 years or older) were selected. Animal studies were excluded.

Each database was searched from the earliest available record up to August 31, 2020. To be eligible for consideration, studies had to meet the following four main criteria: (1) having determined the metabolic rate of at least one energy substrate, either raw or normalized, during endurance exercise lasting from a minimum of $30 \mathrm{~min}$ (to avoid missing lipid oxidation, which is negligible in the early phase of exercise; Spriet, 2014) to a maximum of $120 \mathrm{~min}$ (to avoid ultra-endurance exercise); (2) having tested subjects during aerobic exercise carried out at moderate intensity (between 45 and $65 \%$ of the laboratory-determined peak $\mathrm{O}_{2}$ consumption, according to the American Heart Association Guidelines; Fletcher et al. 2001); (3) having enrolled both healthy men and reproductive-age women, and (4) having reported, compared, and interpreted data based on sex.

Studies conducted on both sedentary/recreationally active subjects and athletic population were considered for this study. However, data were kept separated in the analysis, to avoid heterogeneity.

The initial search was undertaken by three of the authors (AM, GM, MC). The retrieved items were handled using Mendeley Desktop (Version 1.19.5, Mendeley Ltd). The titles and abstracts of the retrieved studies were then independently assessed by three authors (AC, LV, LC); duplicates and records that were clearly ineligible/ out of scope were excluded at this stage. When the title or abstract presented insufficient information to determine eligibility, the full-text papers were evaluated. Based on the information presented in the full manuscripts, eligible studies were included in the qualitative analysis. In cases of disagreement, consensus was reached by discussion and, if necessary, the opinion of a fourth author (AM) was sought (in five occasions) to reach the final decision. When the set of included articles was completed, all their reference lists were manually checked for further relevant publications by three of the authors (AC, LV, LC). Articles including mixed population (i.e., enrollment of both recreationally active and athletes, without reporting data separately) or presenting sex imbalance (e.g., enrollment of more males than females) were not included in the meta-analysis, to control inherent heterogeneity across the studies. 


\section{Assessment of study quality, risk of bias and overall quality of the evidence}

The included studies were assessed independently by three authors (AC, LV and LC) for methodological quality and risk of bias, employing the Study Quality Assessment Tools of the National Institutes of Health (https://www.nhlbi.nih. gov/health-topics/study-quality-assessment-tools). Specifically, the "Quality Assessment Tool for Before-After (Pre-Post) Studies with No Control Group" was employed. This tool consists of a set of 12 criteria in the form of questions covering the main sources of bias. Satisfying $75-100 \%$ or $25-75 \%$ or $<25 \%$ of the criteria is indicative of low, moderate, or high risk of bias, respectively. In case of non-applicable criteria/questions, the total score was calculated out of the highest number of applicable items rather than out of the predefined 12 items. Disagreements between the three authors were resolved by discussion. If consensus could not be reached, the opinion of a fourth author (AM) was sought (in two occasions).

\section{Data extraction process and pre-planned meta-analyses}

A customized data extraction form was developed and applied to each included article by one author (AM) and the extracted data were checked for accuracy by a second author (LC). The extracted data included information regarding the participants (e.g., sex ratio, fitness level, anthropometric characteristics, oral contraceptives use, dietary habits), the pre-testing condition and exercise protocol (e.g., pre-testing dietary conditions, menstrual phase, duration, intensity relative to peak $\mathrm{O}_{2}$ consumption, exercise modality-e.g., walking, cycling, etc.), outcome measures (i.e., raw or normalized as percentage), main findings (e.g., carbohydrates oxidation: men $>$ women).

Based on the state of the art, we predefined a set of sexcomparative meta-analyses of percent and raw data for the following variables: carbohydrate oxidation (including, but not limited to, muscle glycogen and glucose utilization, rate of appearance and disappearance); lipid oxidation (including, but not limited to, FFA and IMCL); protein oxidation (including, but not limited to, amino acid utilization and disposal). To control for heterogeneity deriving from inconsistencies in the training status, we performed separate analyses for sedentary/recreationally active and athletic populations.

\section{Thematic analysis of the mechanisms mediating sex-based differences}

To gather mechanistic insights into the possible physiological correlates of the observed sex differences in substrate utilization, a thematic analysis was performed. Each individual study was carefully read to outline relevant investigated and/or suggested physiological mechanisms. Original text extracts (direct quotes) were then obtained, and recurrent concepts were highlighted and subsequently coded (e.g., "adrenergic mechanism: receptor type and catecholamines levels" or "adrenergic regulation of lipid mobilization"). Single themes that could gather several codes (e.g., "adrenergic activation") were generated a posteriori by consensus among the three authors (AC, AM, LV). Themes were then highlighted within each paper and used to qualitatively appraise the mechanisms investigated and/or suggested by the authors. In case the authors tested or proposed more than one mechanism, only those for which sex-based differences emerged were computed. Mechanisms associated to both fat and carbohydrate metabolism were considered separately. If two or more mechanisms were found/suggested to mediate the observed differences between men and women, the hierarchical order of importance drawn by the authors was followed.

\section{Data analysis}

A meta-analysis was performed if at least three studies reported data for the same outcome measure. RevMan 5.4.1 software (Review Manager, The Cochrane Collaboration; 2020) was used to aggregate the extracted data and to obtain pooled estimates of the difference between men and women. Raw data (means and standard deviations, SD) were extracted or calculated from other statistics reported in the paper (i.e., standard error; 95\% confidence interval, CI). If studies reported outcomes exclusively through graphs, the mean scores and the related measures of spread (SD, standard error, 95\% CI) were estimated employing GetData Graph Digitizer (version 2.26.0.20). A random-effects model was chosen for all meta-analyses to account for potential methodological differences in the assessment and training protocols across studies, as conventionally done in biomedical research (Borenstein et al. 2010). To allow interpretation of the pooled estimate of an effect, the weighted mean difference (MD) with $95 \%$ CI was calculated when pooling data from an outcome measure that was homogeneously assessed across studies, whereas the standardized mean difference (SMD) was calculated when the extracted data for one outcome were expressed with different measurement units, or when different testing protocols or exercise modalities (e.g., treadmill walking, over ground walking, cycle ergometer) were employed. Additionally, to estimate the magnitude of the effect size through a standardized index, the SMD was reported for all MD (taking an SMD of 0.2 as small, 0.5 as moderate, and 0.8 as large). In both cases, the level of significance was set at $p<0.05$, as conventionally done in meta-analyses. Heterogeneity across the studies was evaluated using the Chi-square and the inconsistency $\left(\mathrm{I}^{2}\right)$ test; 
a value $>50 \%$ was considered indicative of significant heterogeneity (Higgins et al. 2003). In case of heterogeneity exceeding this threshold, a leave-one-out sensitivity analysis was performed to check whether our findings were driven by a single study.

For those comparisons in which data were obtained from at least ten studies (Sterne et al. 2011), publication bias was assessed by visual inspection of funnel plot asymmetry. To evaluate differences in methodological quality between the studies conducted in sedentary/recreationally active and athletic populations, the Mann-Whitney $U$ test was performed. As for all the other comparisons, the significance level was set at $p<0.05$.

\section{Results}

\section{Selected articles}

The search strategy identified 1077 potentially relevant records (from PubMed/Medline, 362 records; Scopus, 381 records; Web of Science, 334 records). After merging the items retrieved from the databases, duplicates were removed leaving 463 unique articles. Of these, 405 were discarded based on title and abstract, whereas 58 were assessed in full text. Thirteen studies, which did not satisfy the predefined inclusion criteria, were excluded. The remaining 45 studies, deemed eligible, were included in the qualitative analysis. Figure 1 presents the flowchart of the study selection process. The main features of the 45 studies included in the qualitative analysis are summarized in Tables 1 and 2 (i.e., participants' status, pre-testing conditions and employed exercise protocols) and Tables 3 and 4 (i.e., outcome measures, main findings and suggested physiological mechanisms), in sedentary/recreationally active (28 studies) and athletic (17 studies) populations, respectively.

\section{Quality of the included studies}

Tables 1 and 2 also report the results of the analysis of the methodological quality and risk of bias for the included studies, as assessed by the NIH Study Quality Assessment Tools. Three items proved not applicable to the design of the studies considered for the present study (item 9: losses to follow-up after baseline; item 11: multiple assessments before and after the intervention; item 12: use of individual-level data); therefore, the score was calculated out of 9 rather than 12 items. The mean score of the 28 studies on

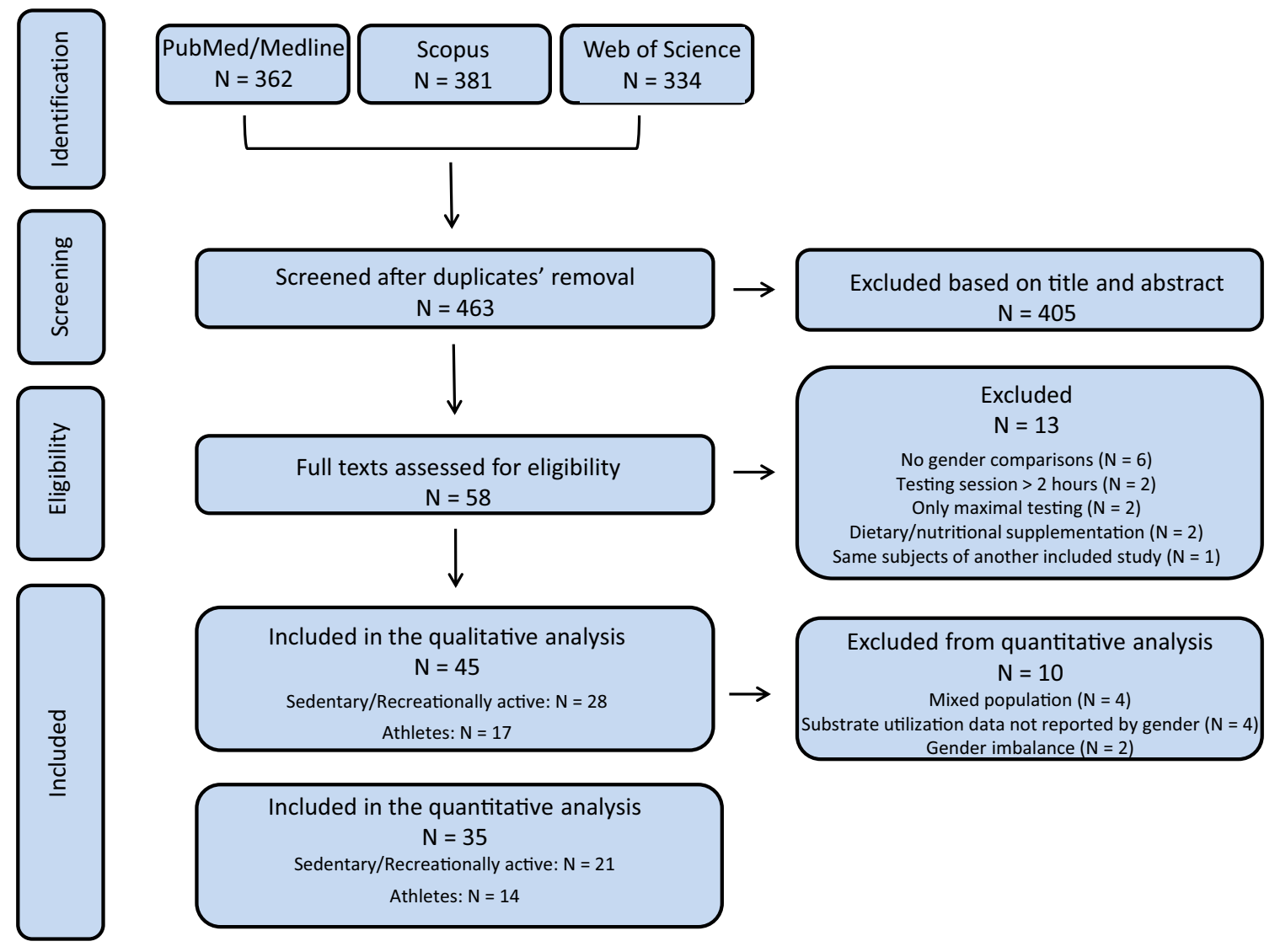

Fig. 1 Flowchart of the studies 


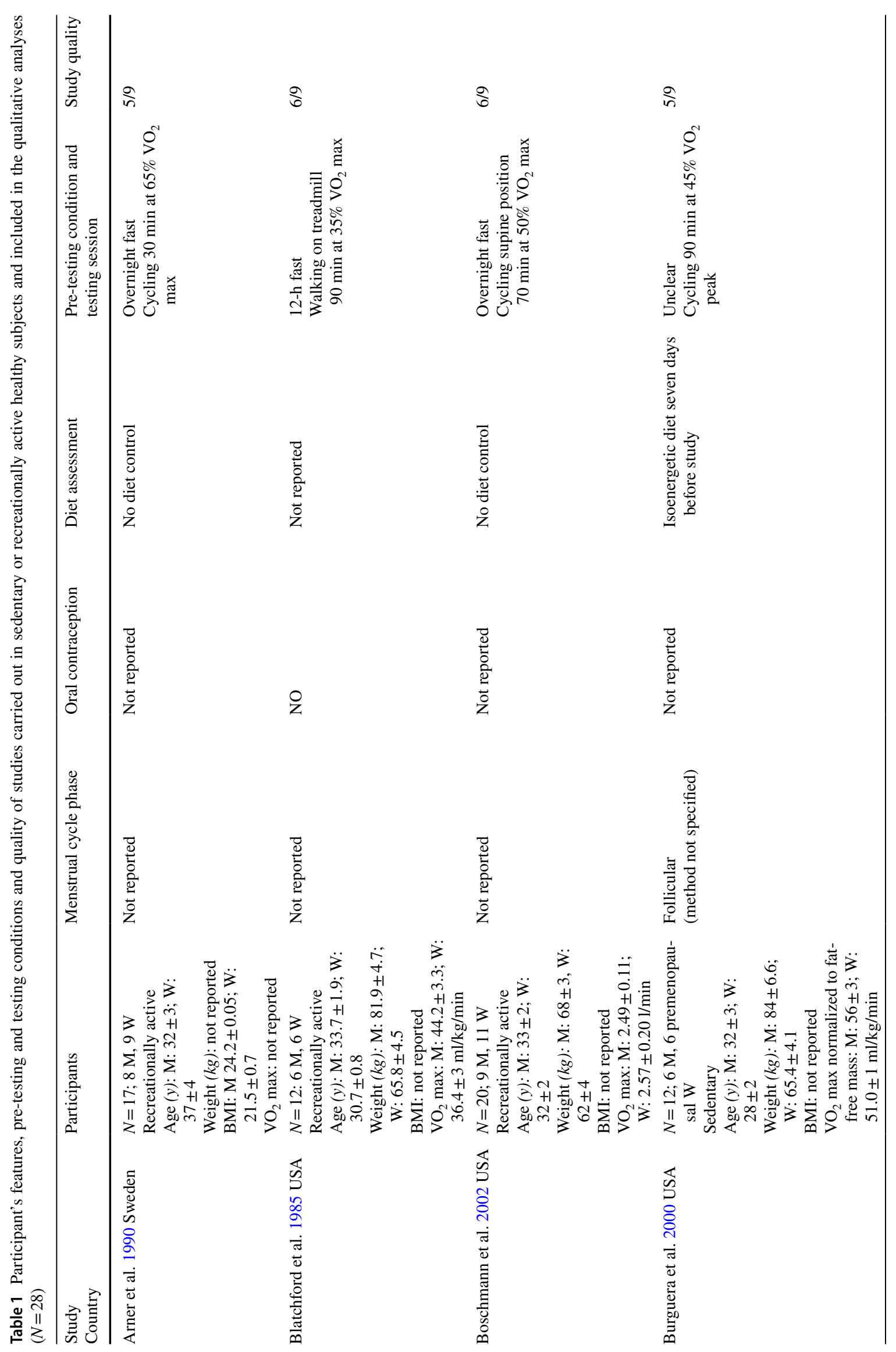




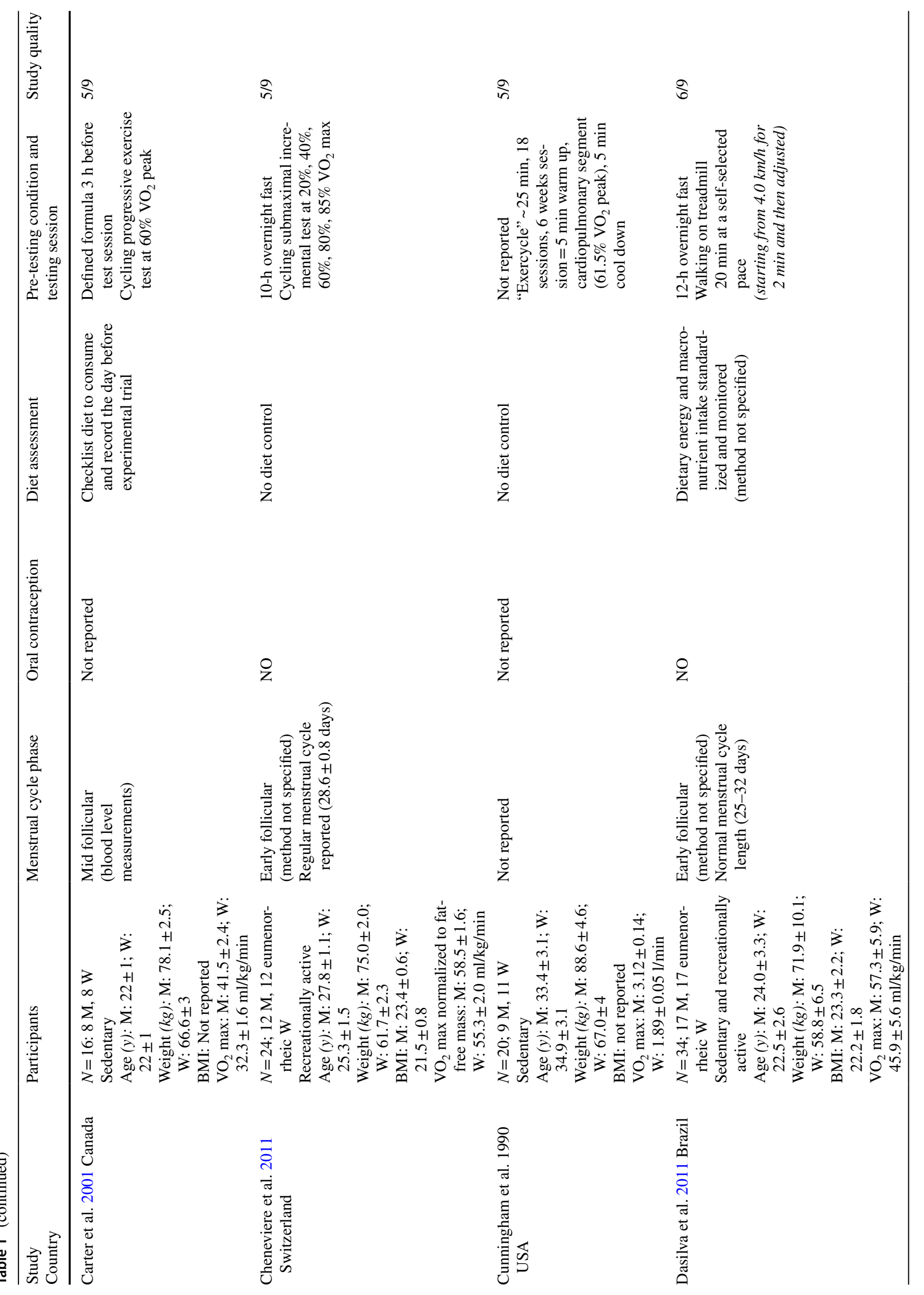




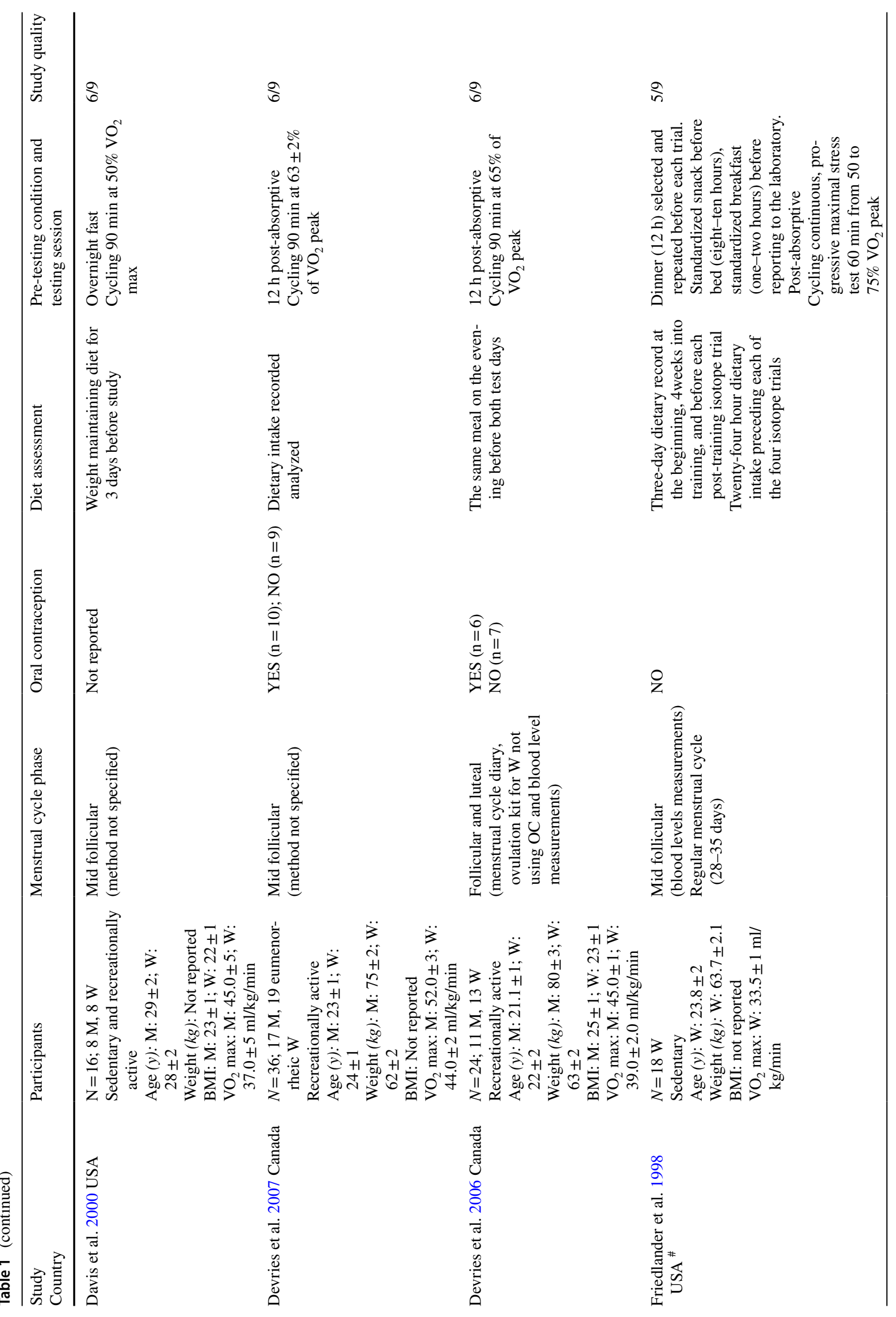




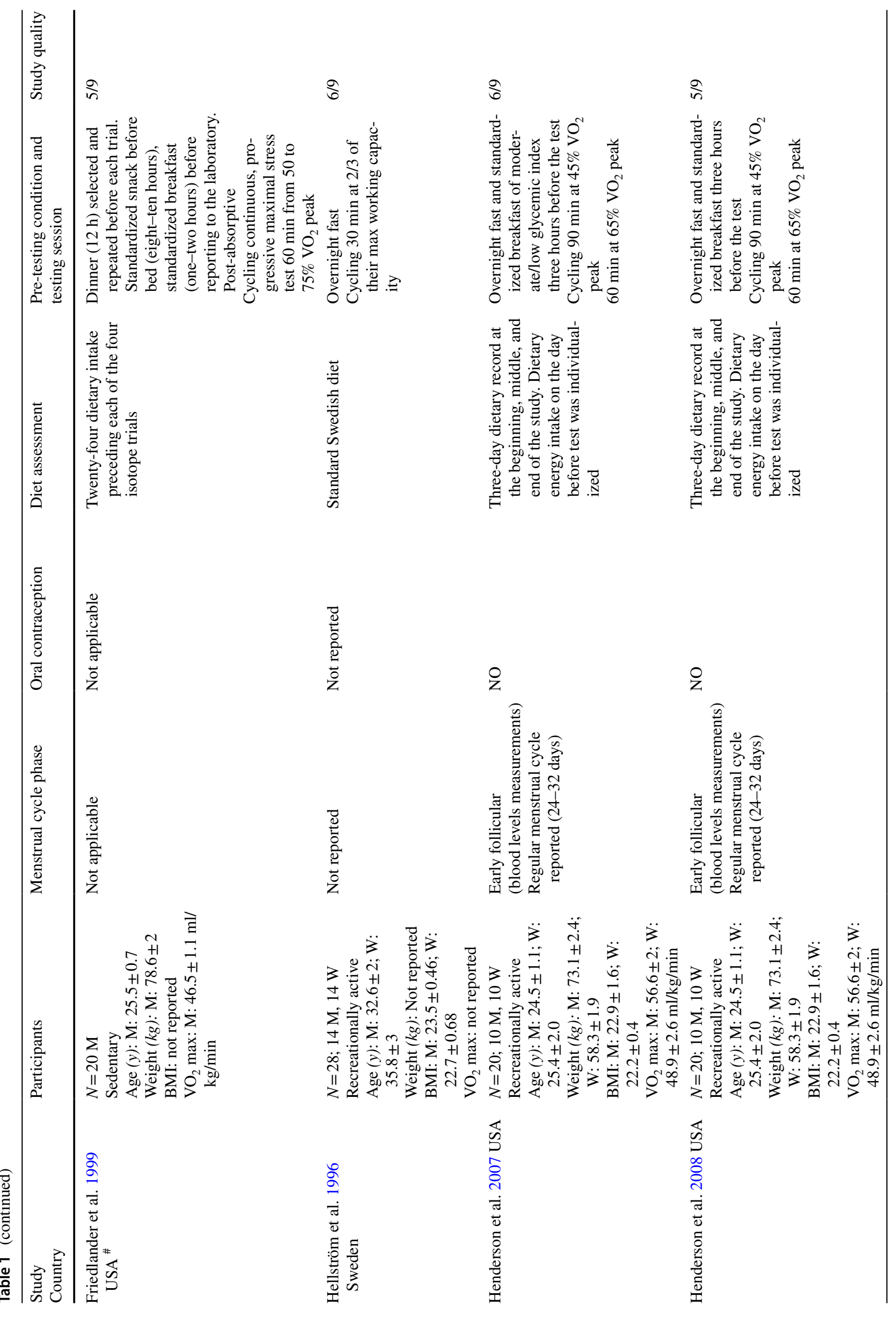




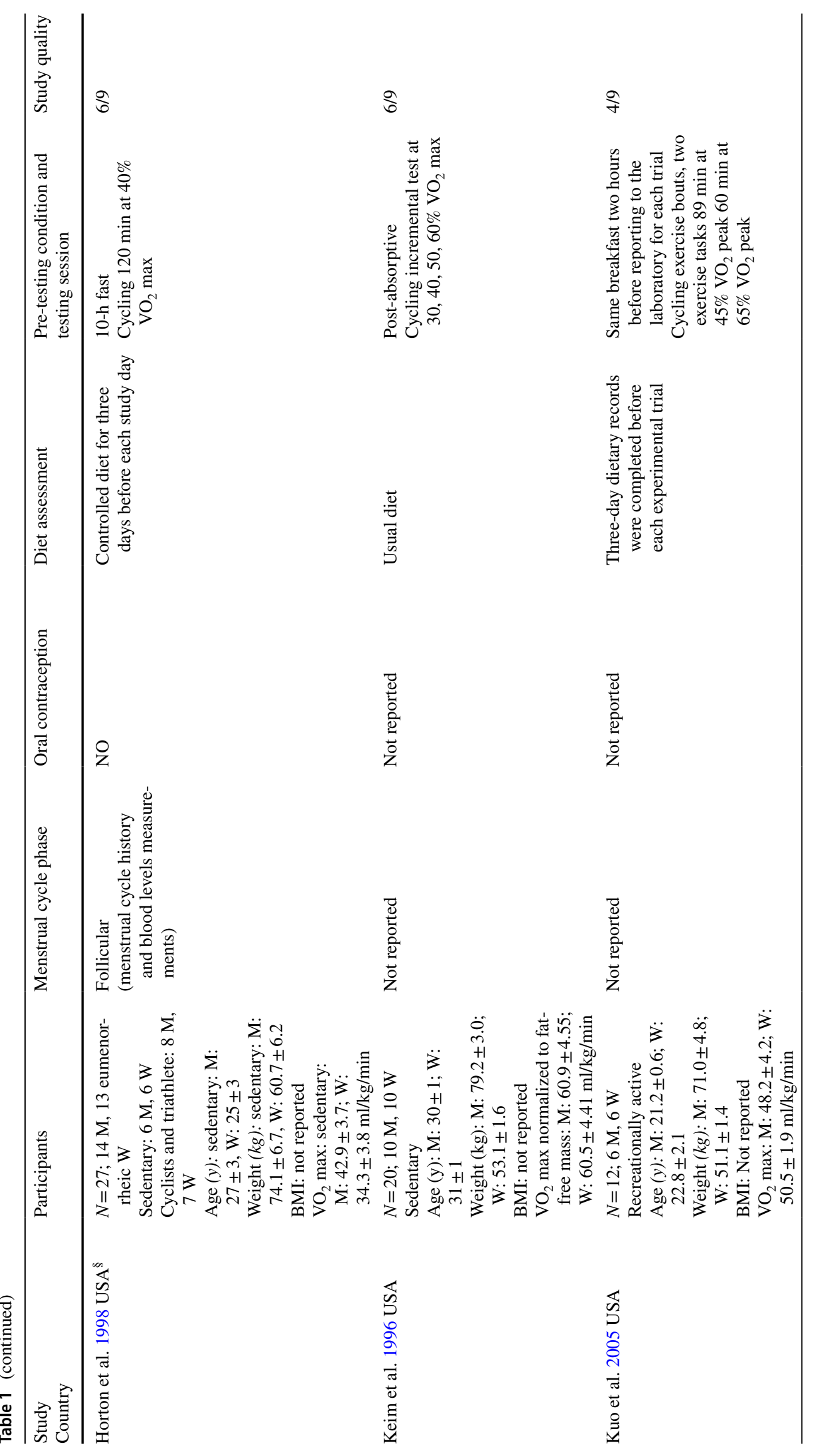




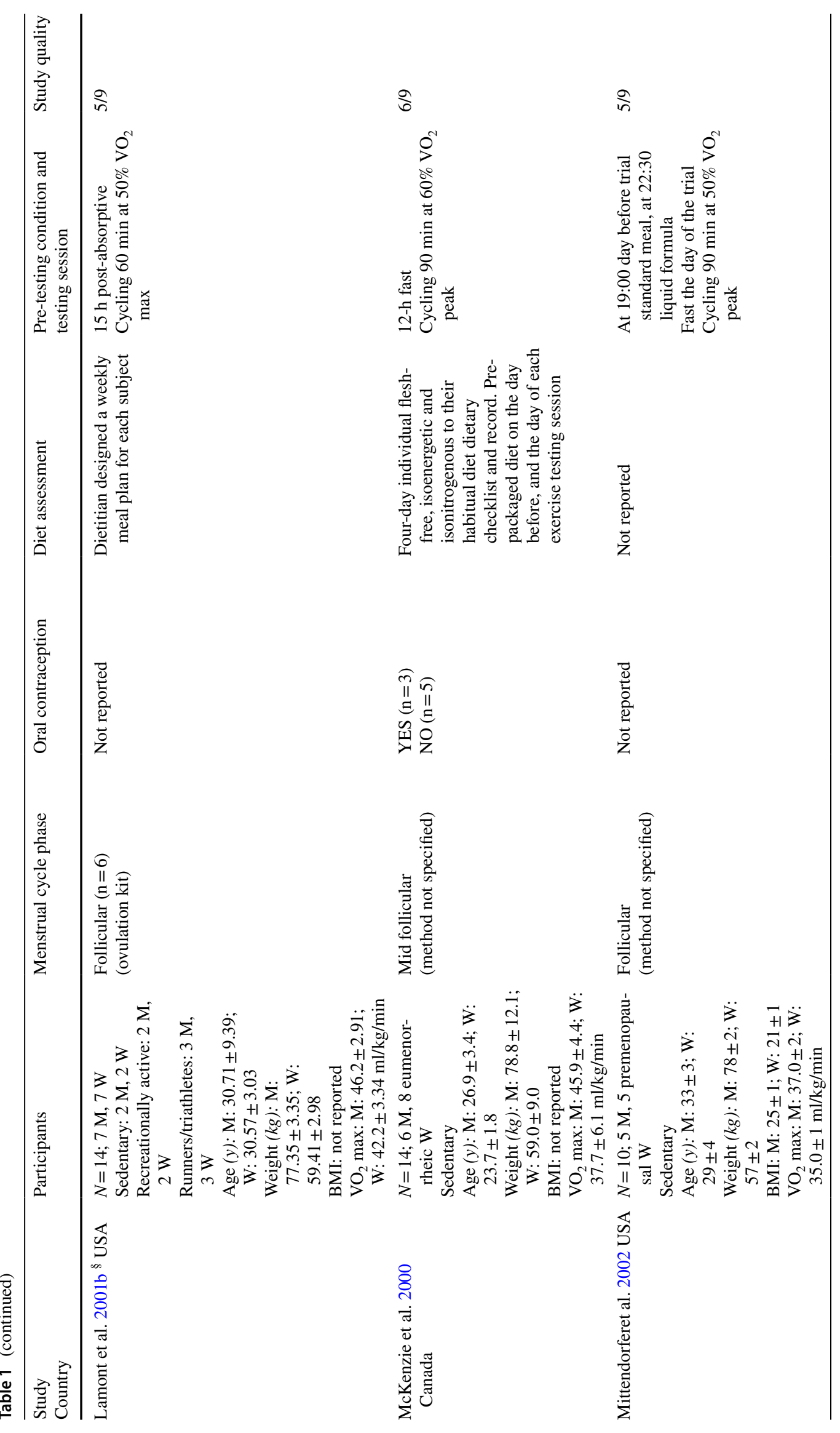




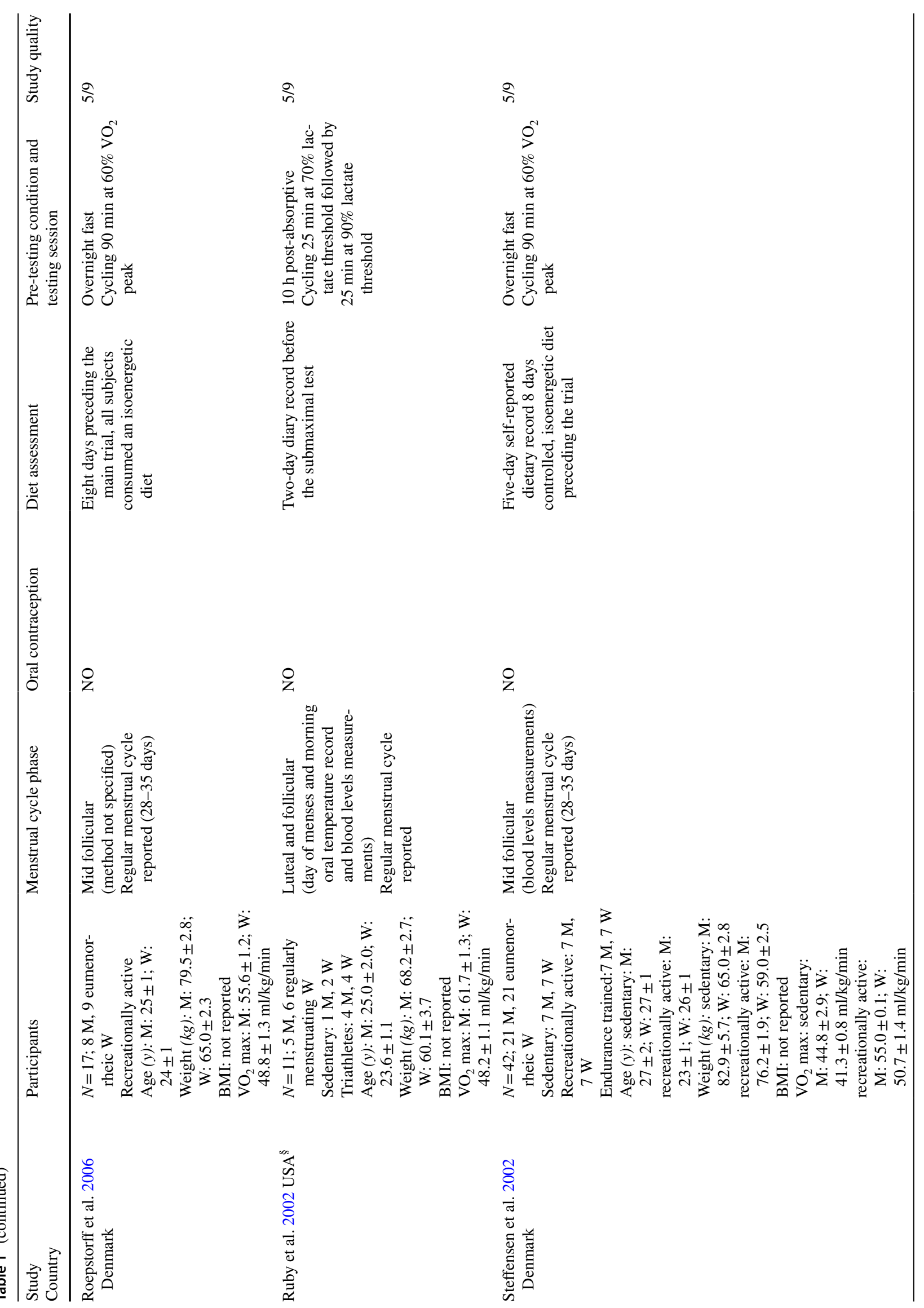




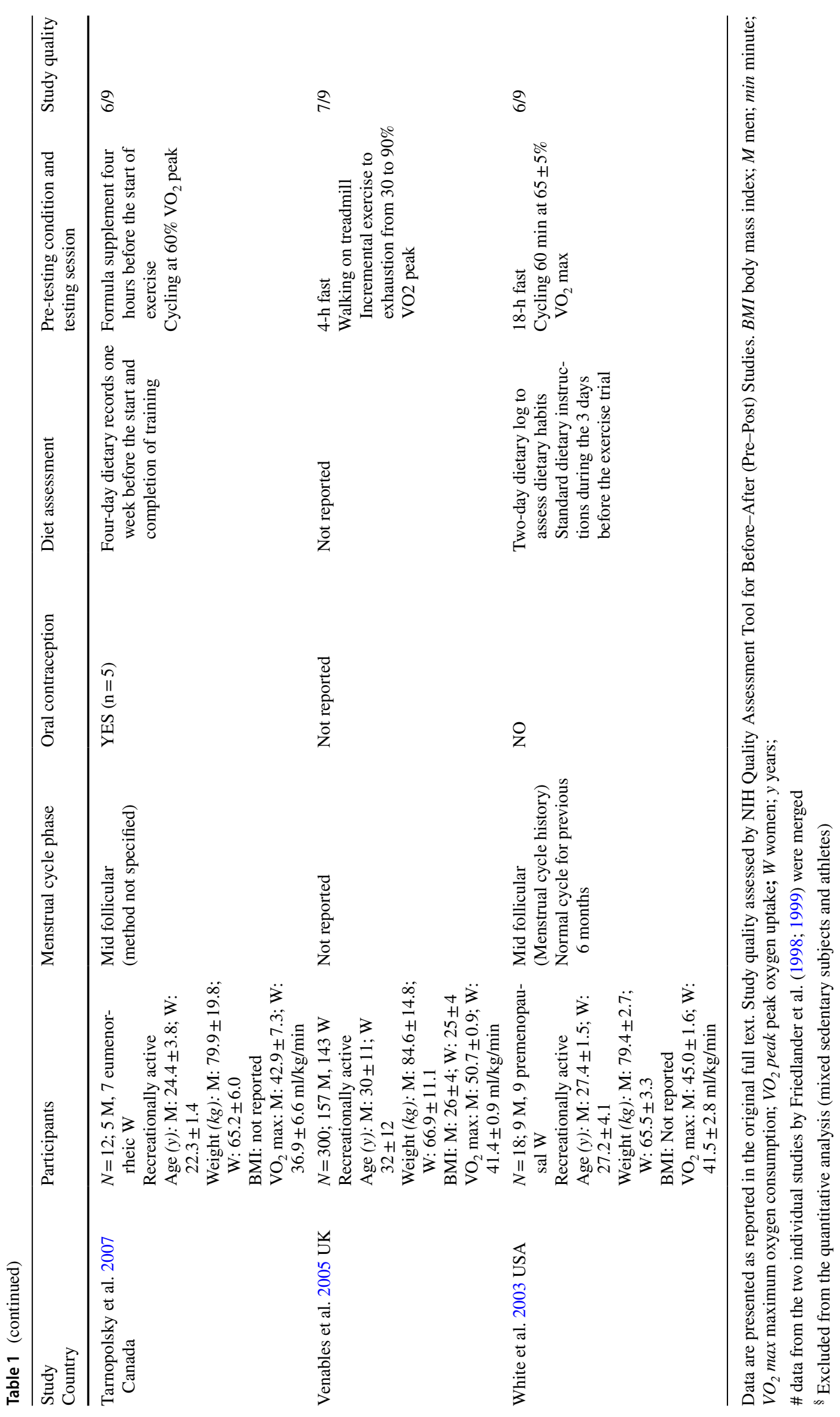




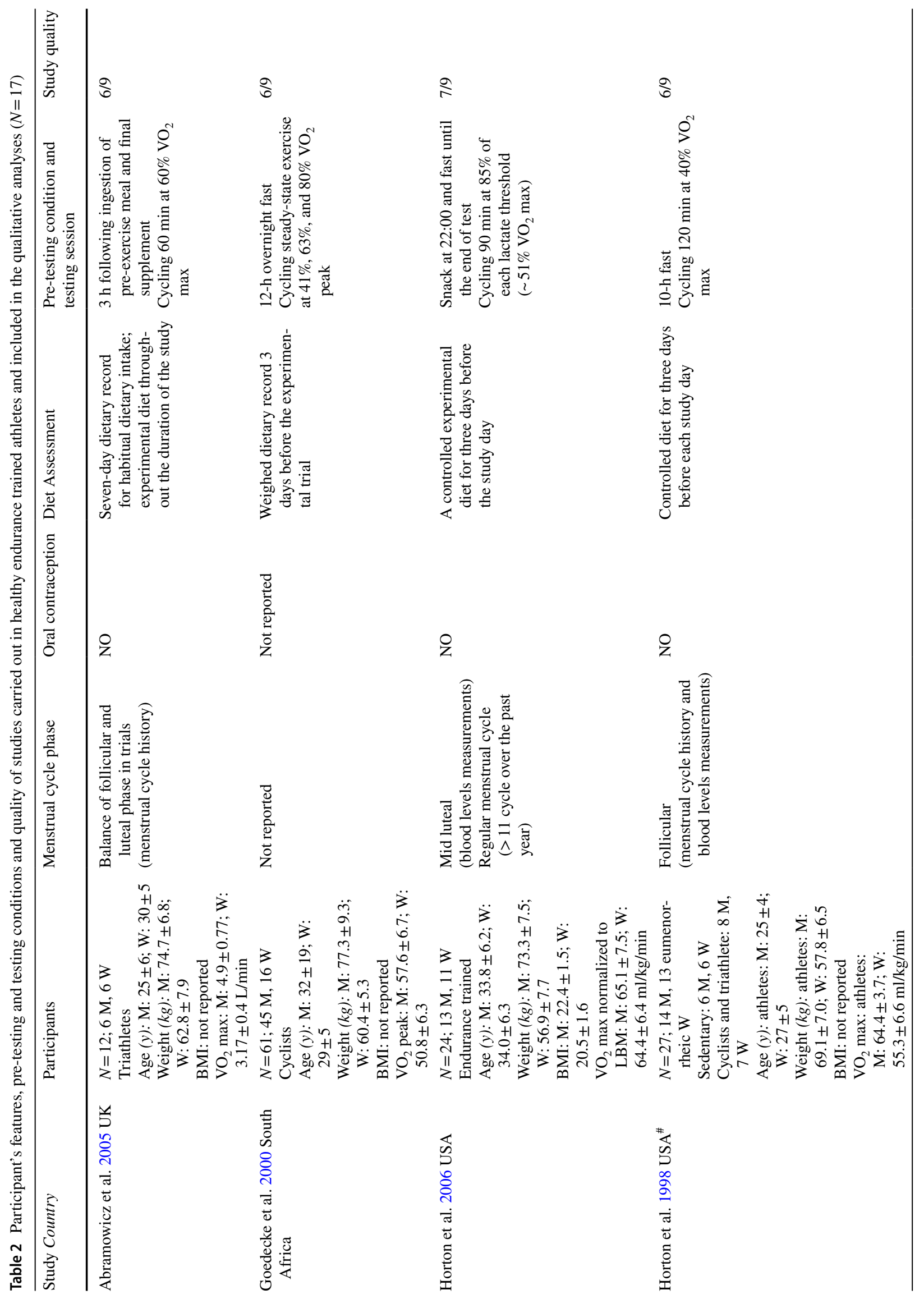




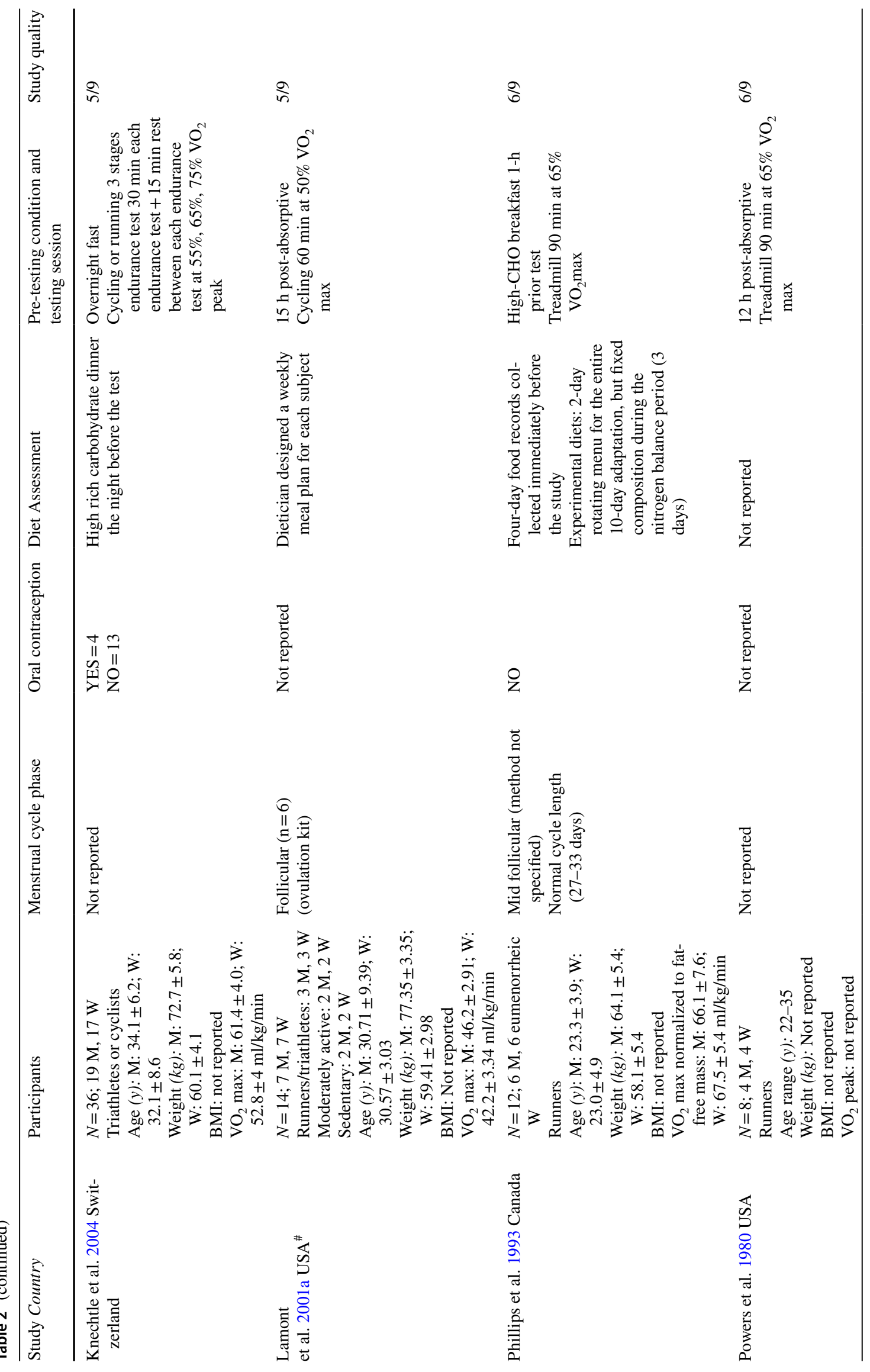




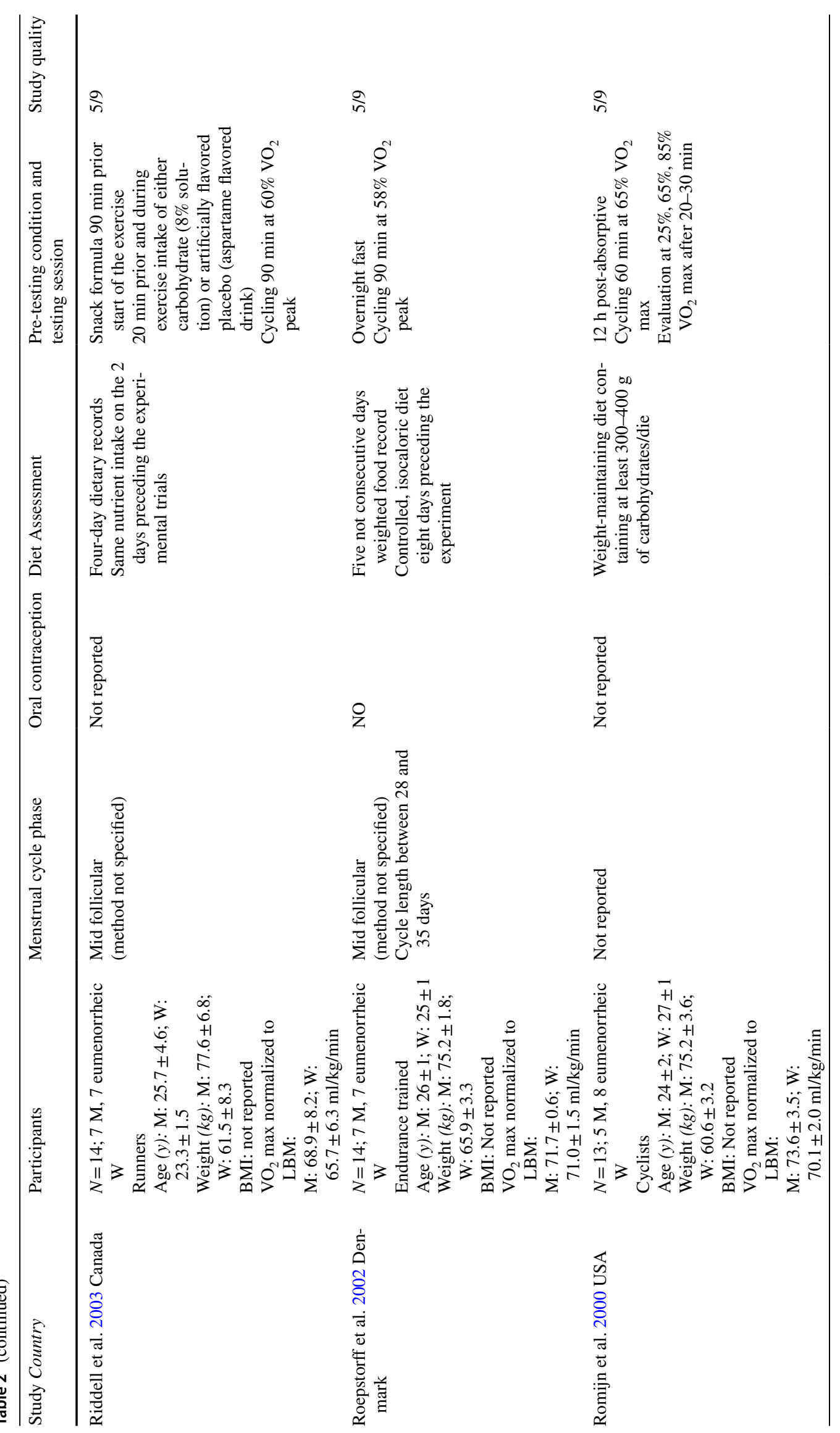




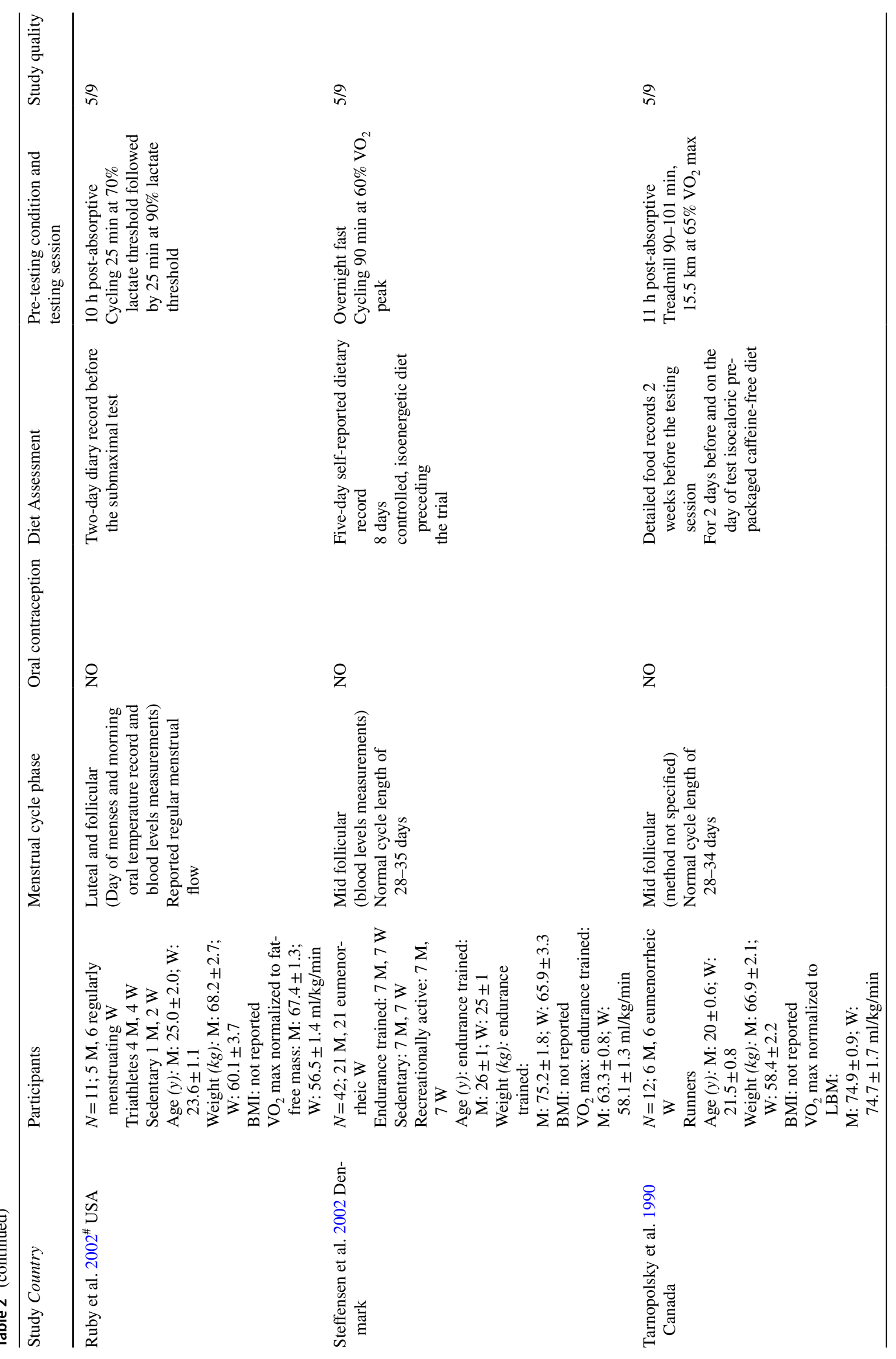




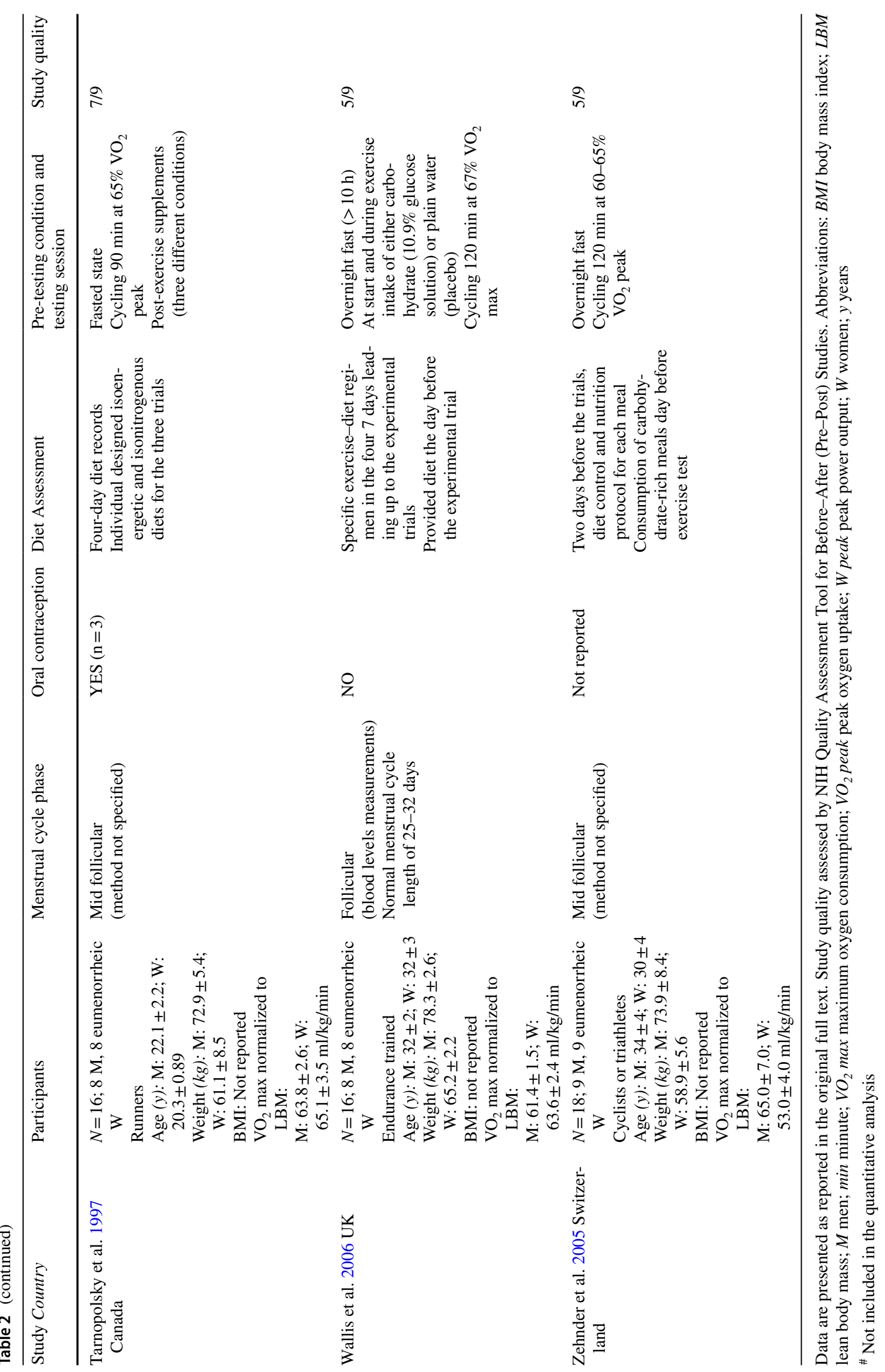




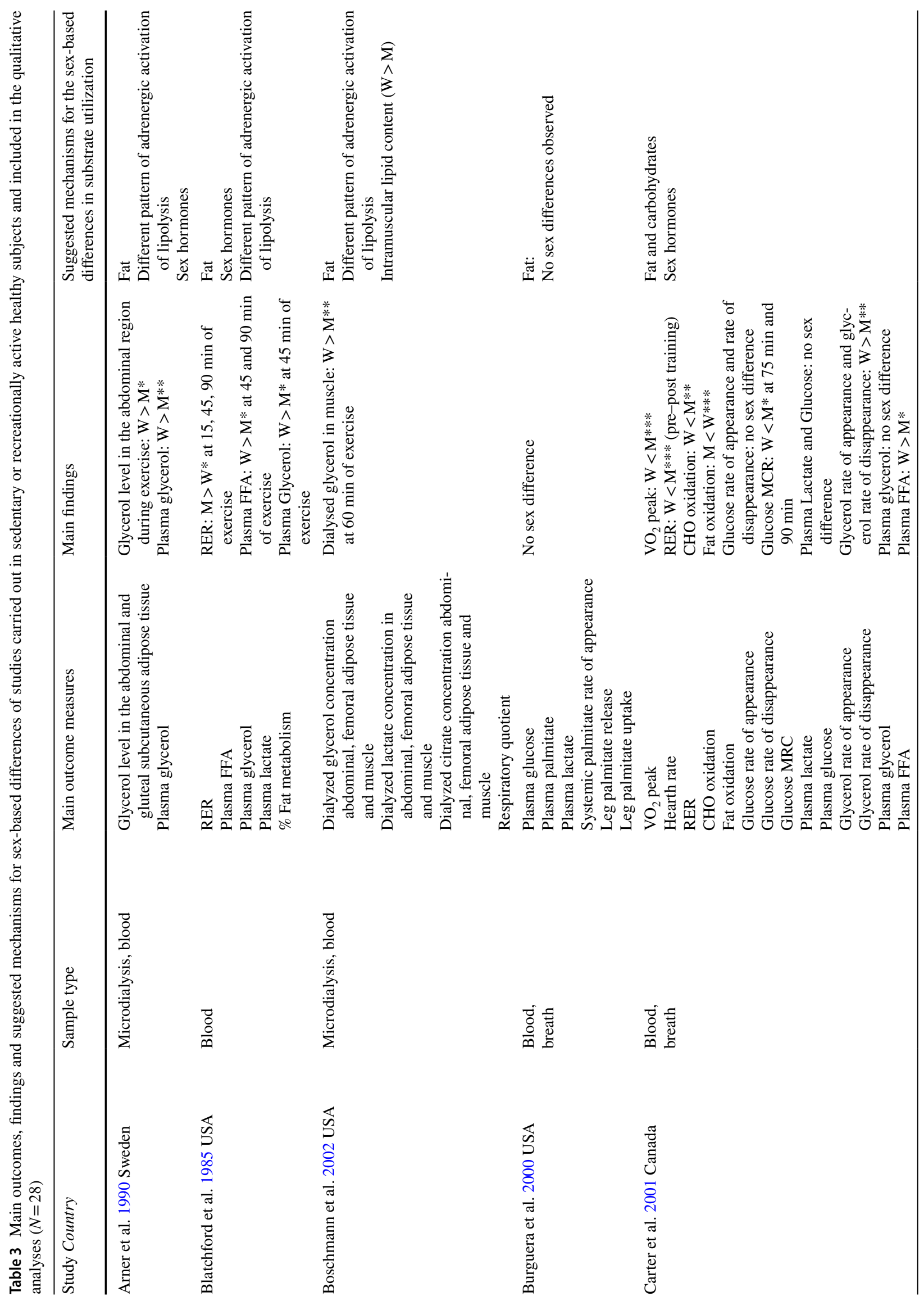




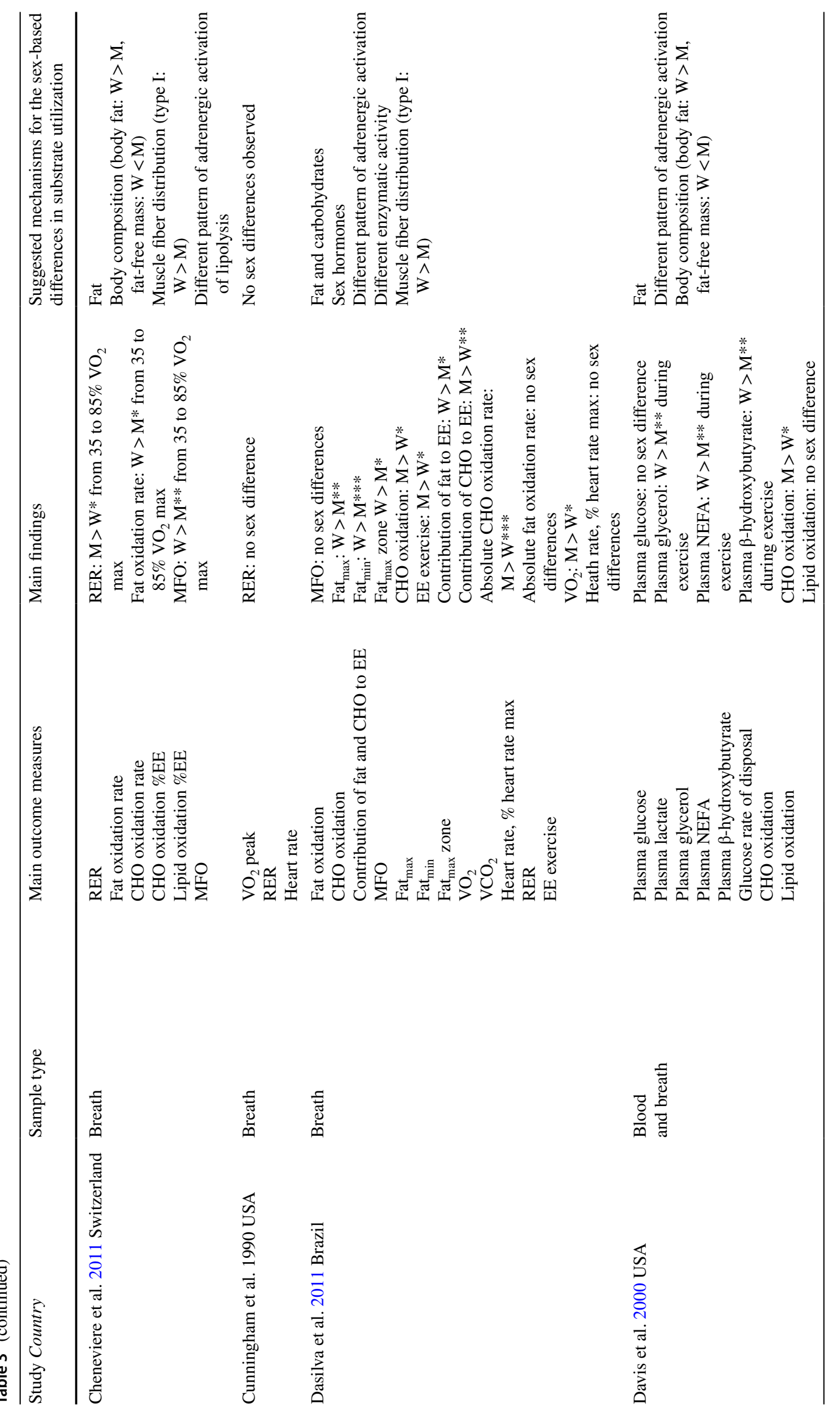




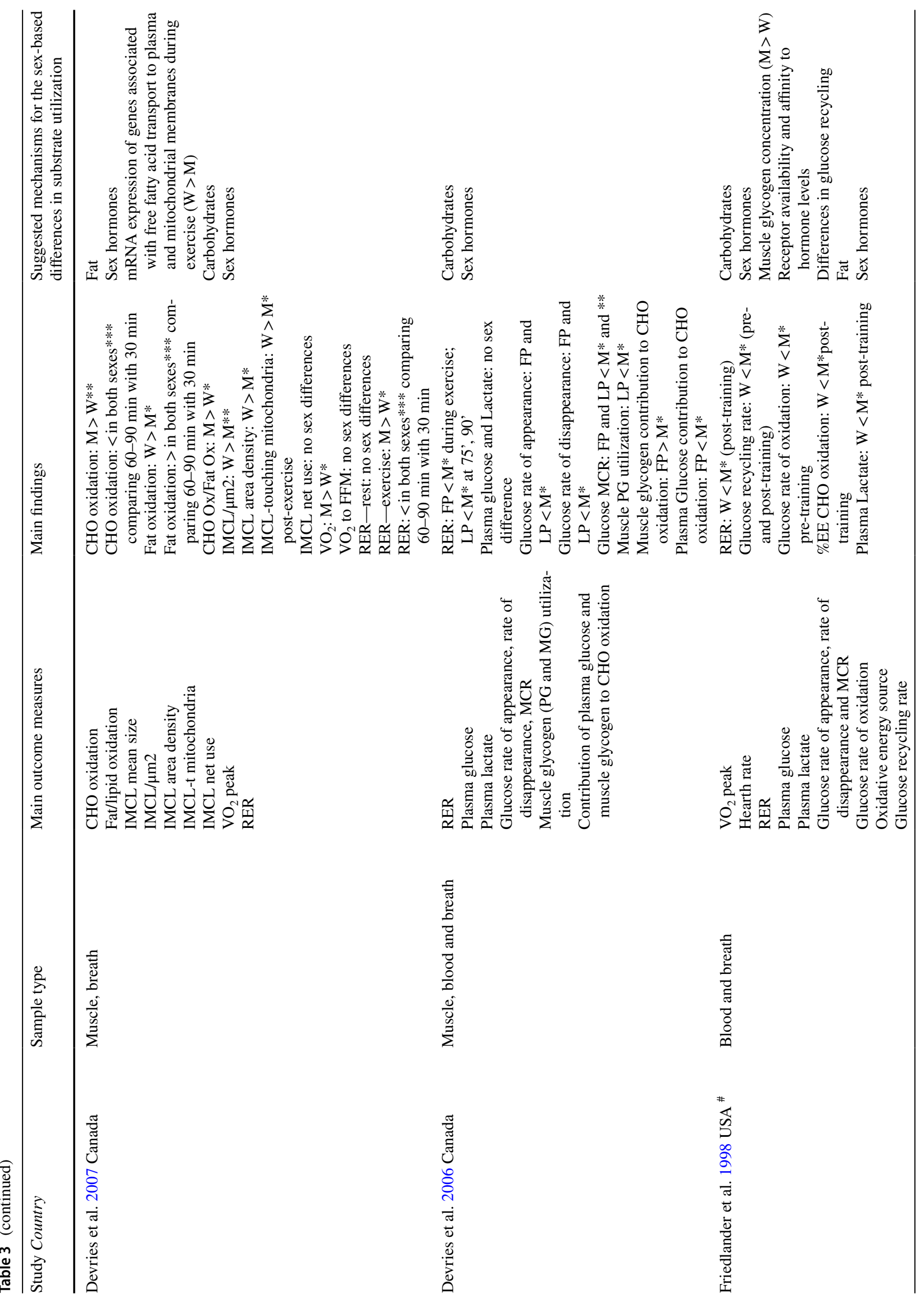




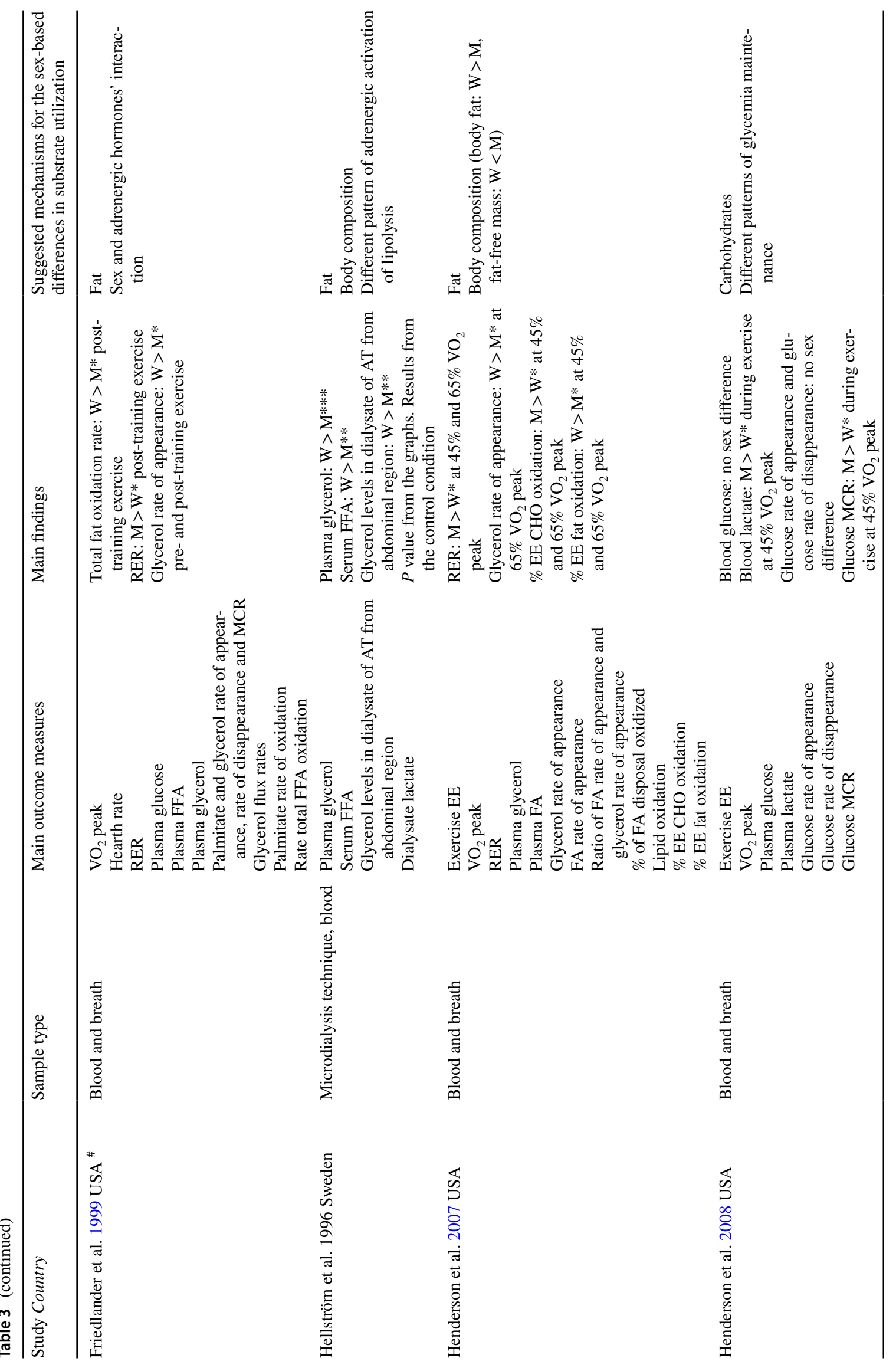




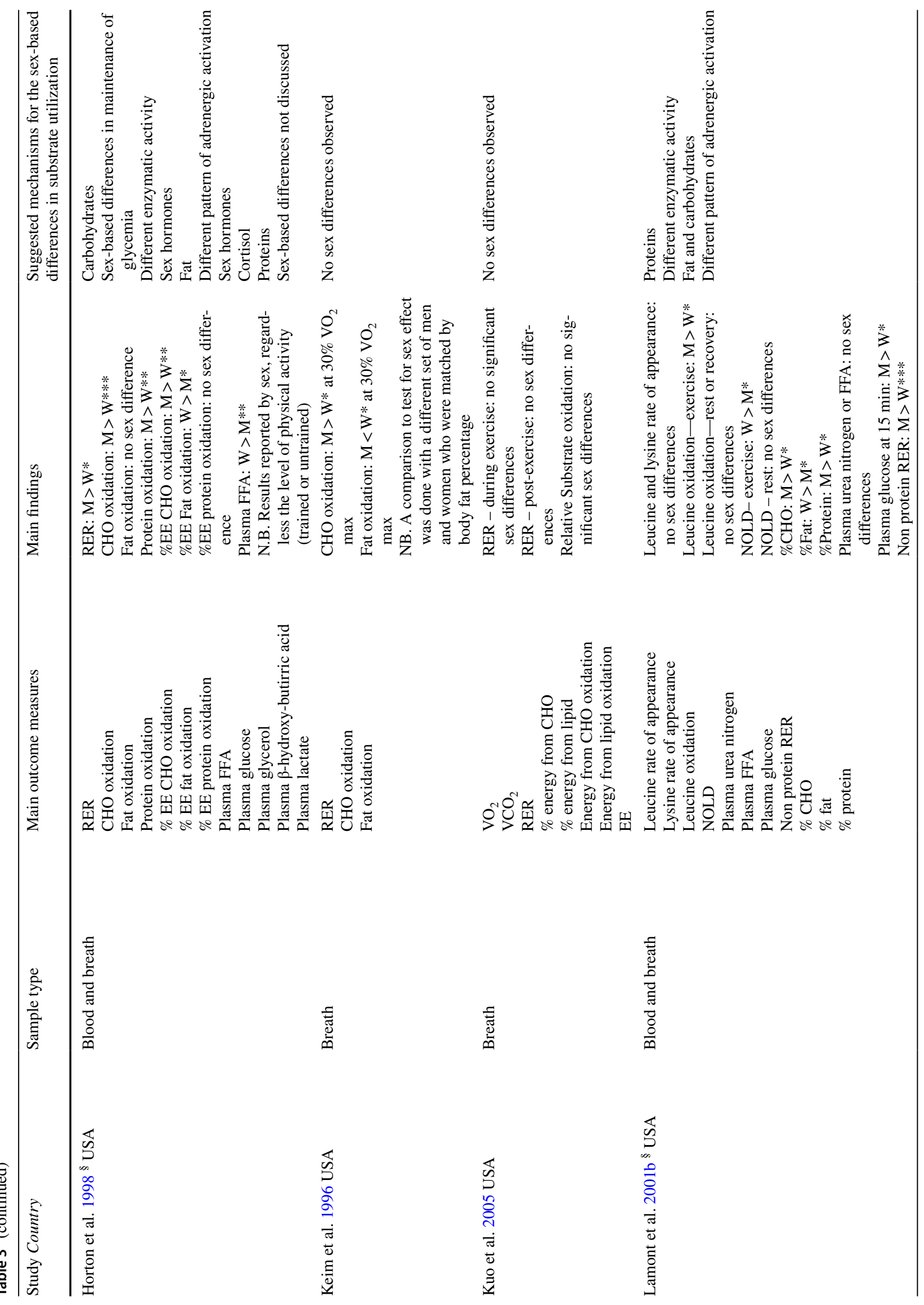




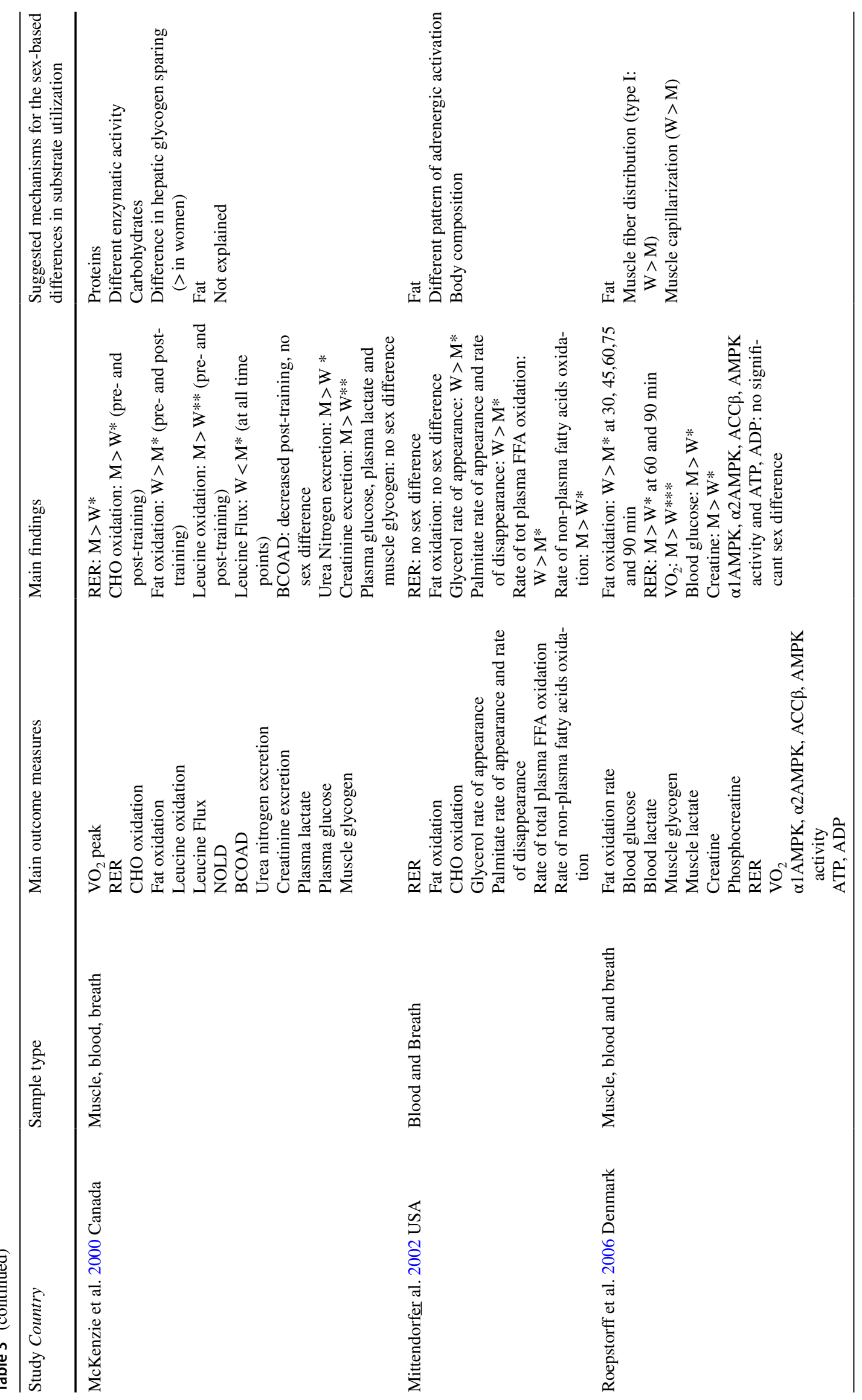




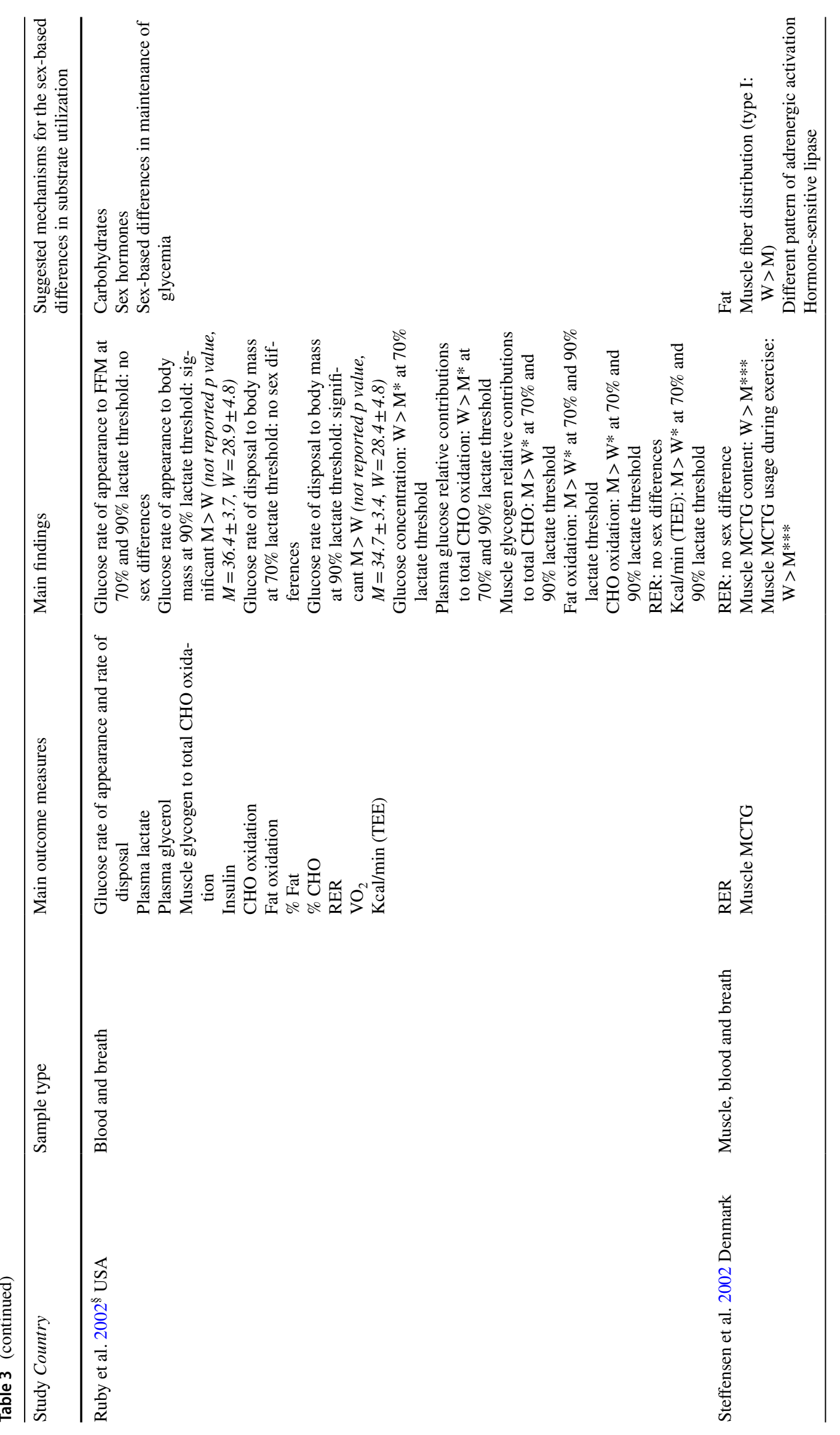




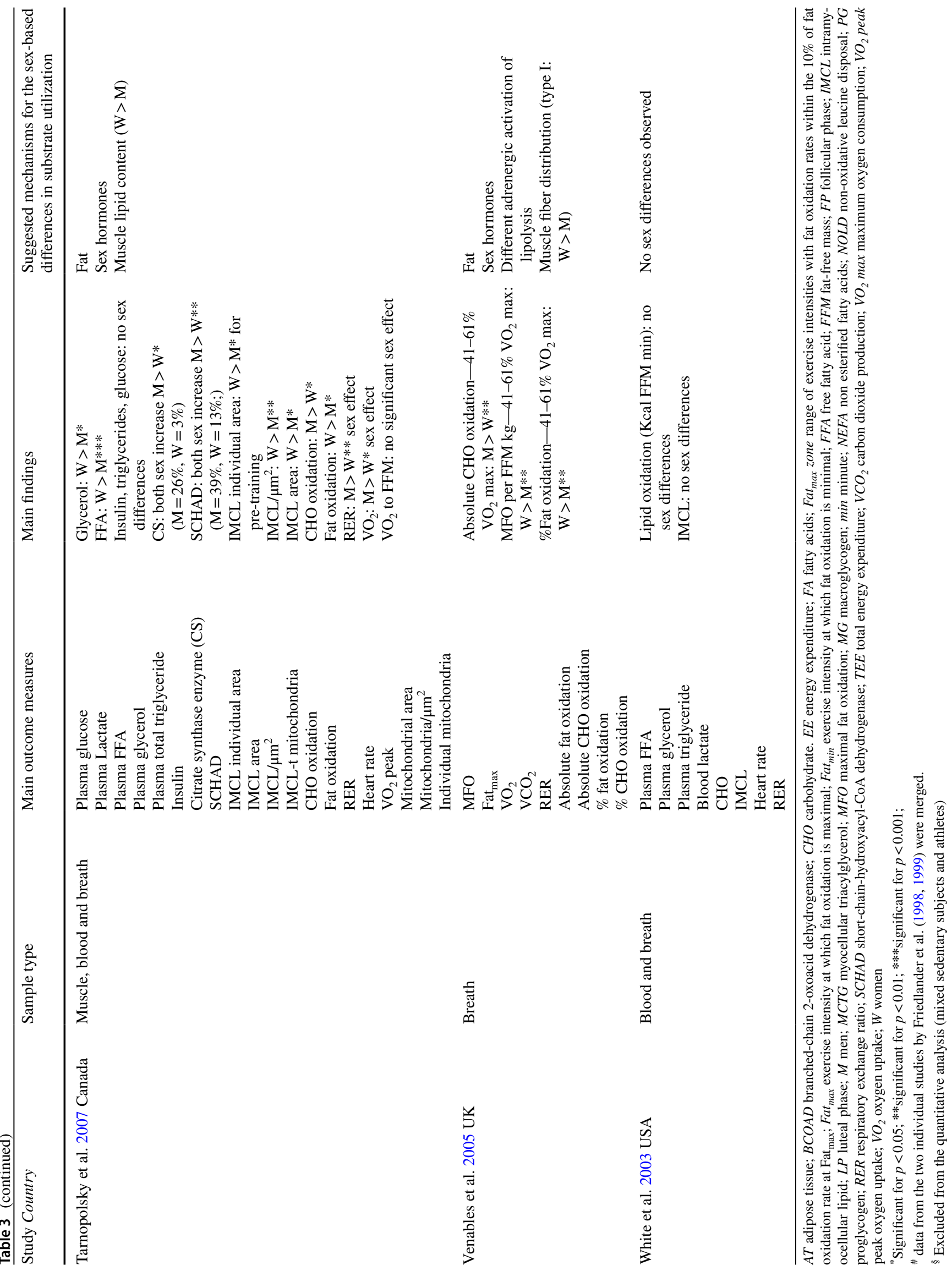




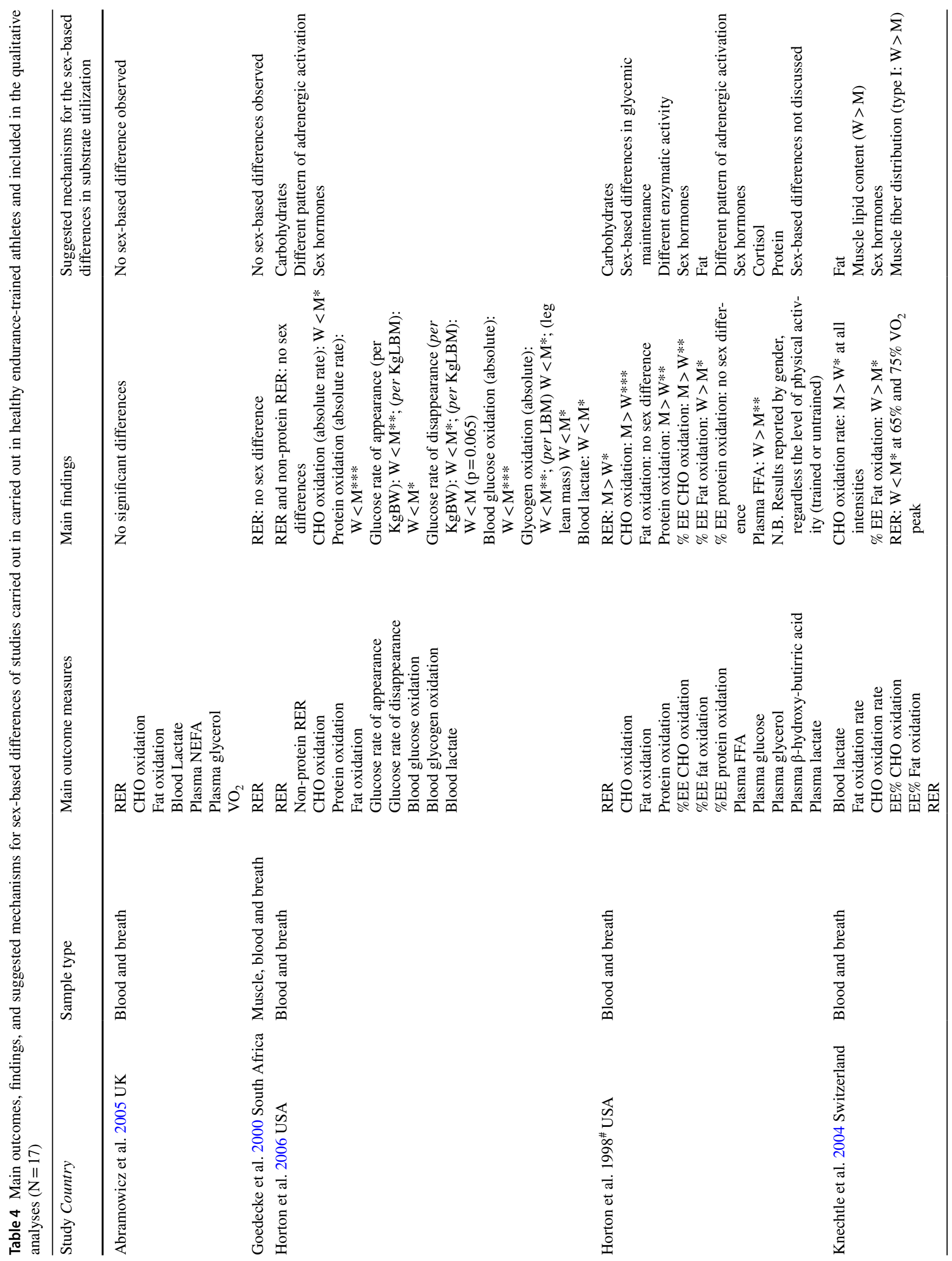




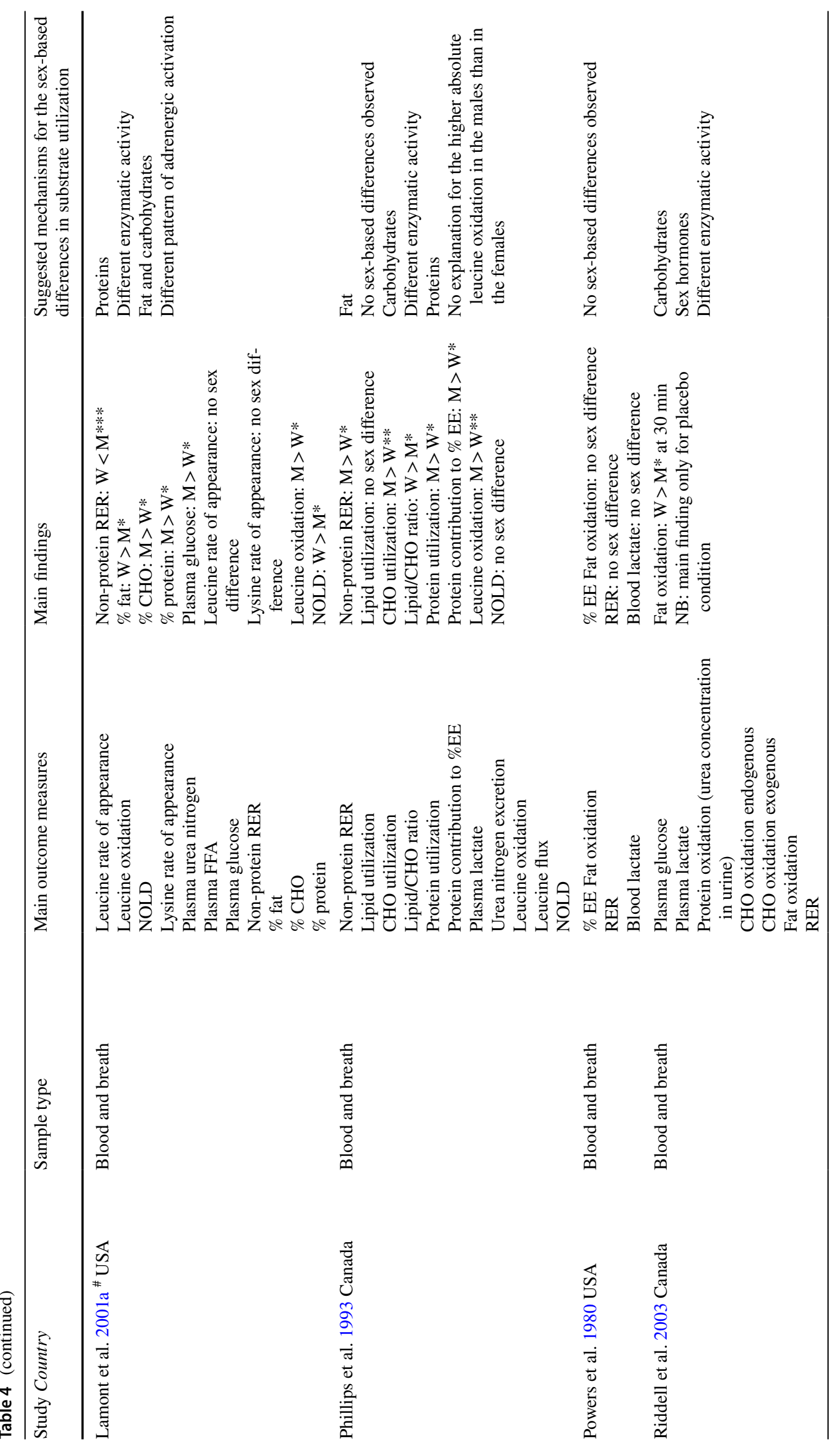




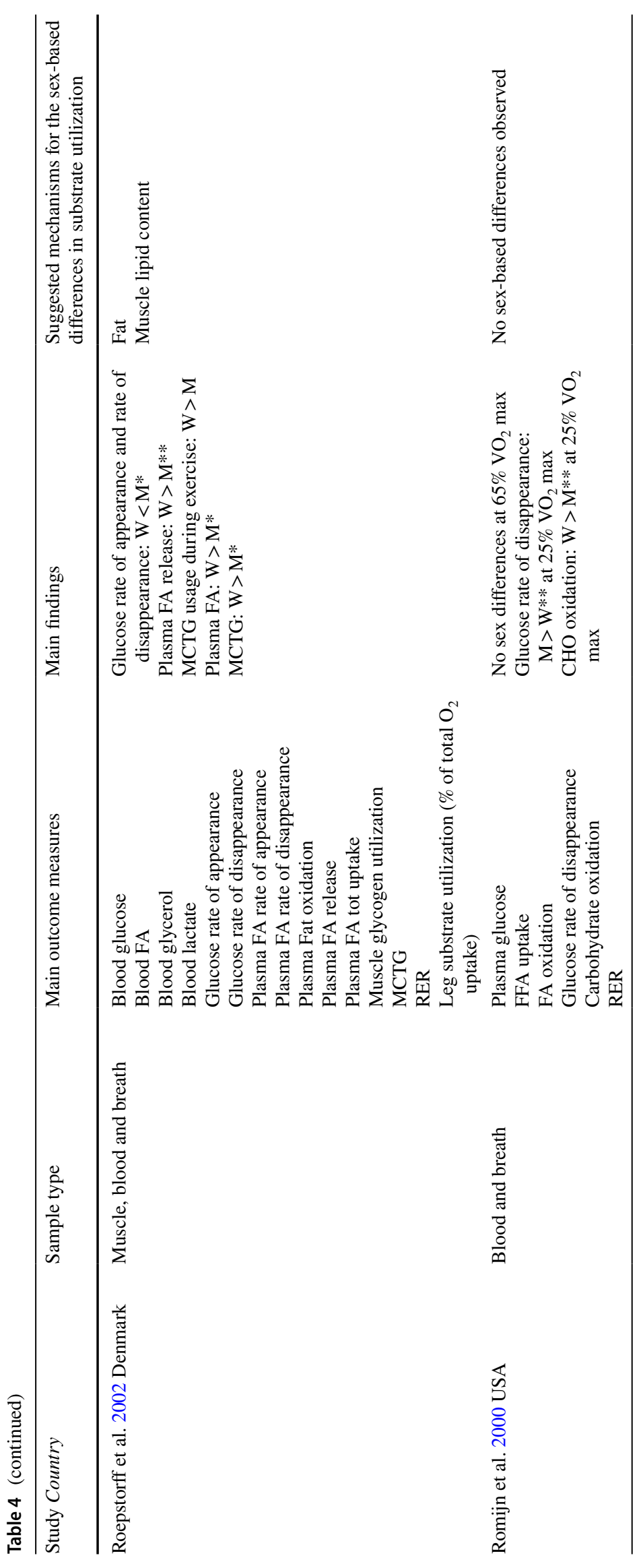




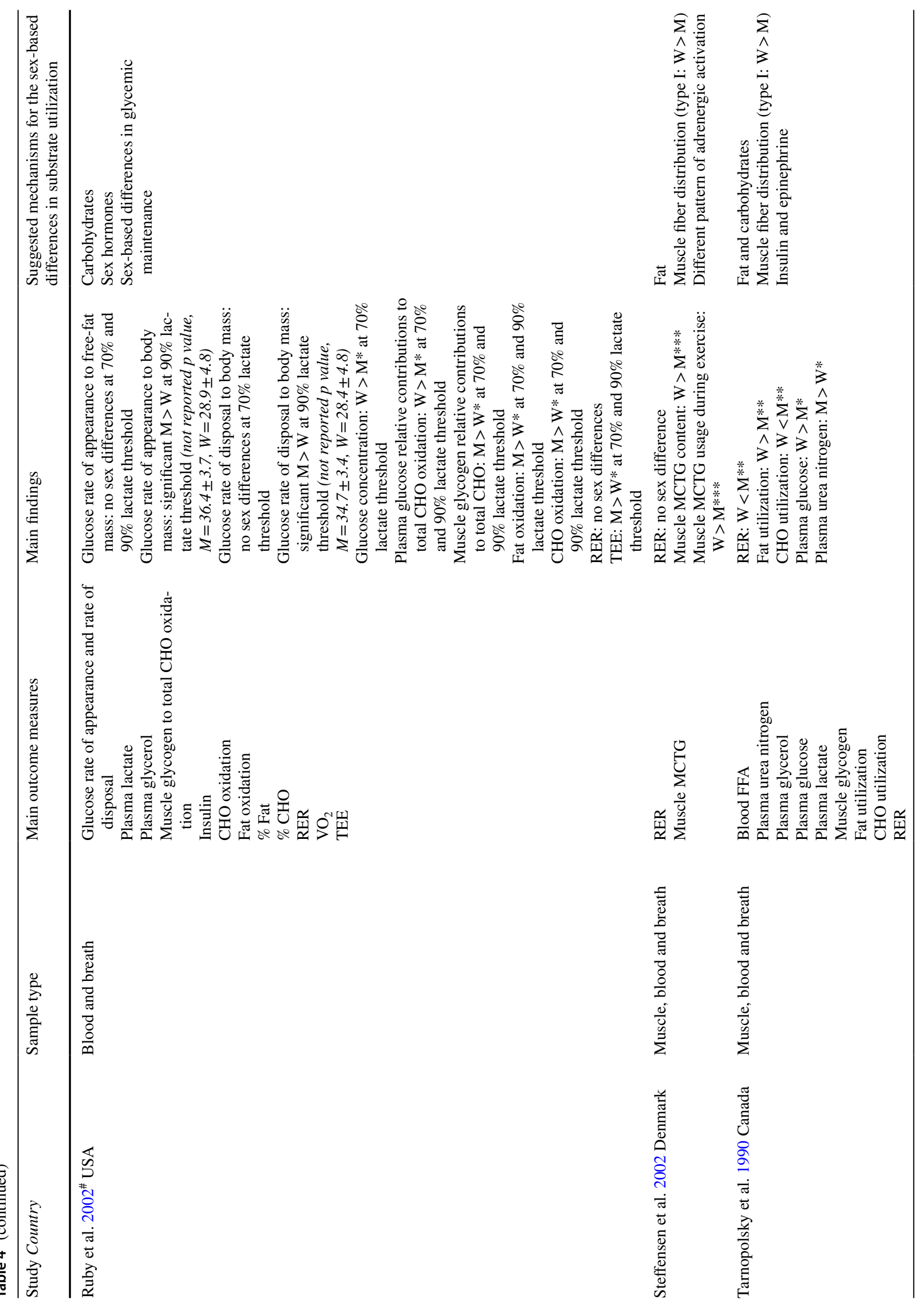




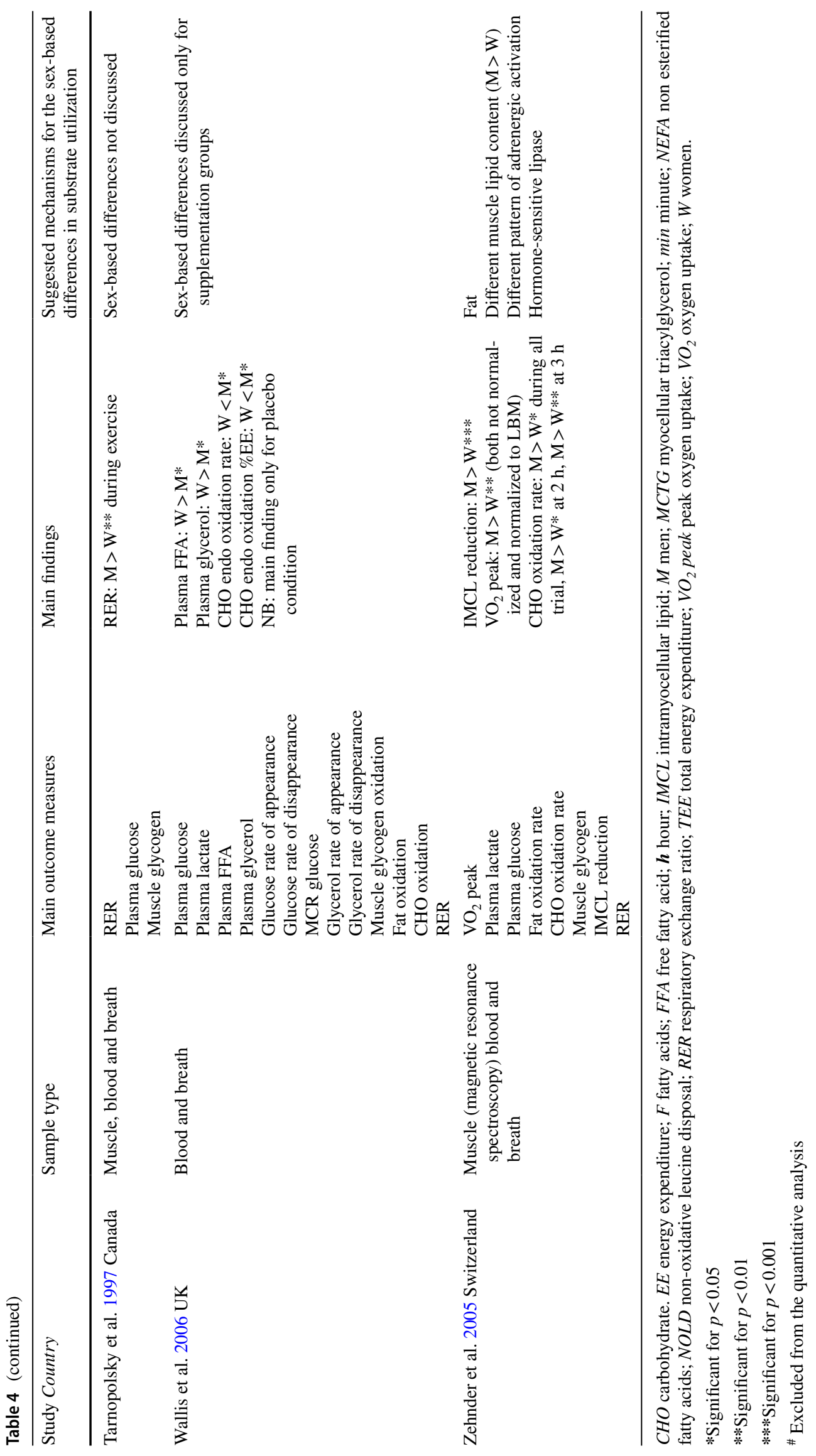


sedentary/recreationally active subjects was $5.5 \pm 0.64$ (95\% CI: 5.29 to 5.79; median: 5.5). The 17 studies on athletes/ highly trained subjects had an average score of $5.53 \pm 0.72$ (95\% CI: 5.16 to 5.90; median: 5.0). The Mann-Whitney $U$ test revealed no significant difference between the scores of the two study groups $(p=0.81)$. In both cases, the most frequently unsatisfied criteria were items 3 (12 out of 45 studies; "Were the participants in the study representative of those who would be eligible for the test/service/intervention in the general or clinical population of interest?"), 4 (8 out of 45 studies; "Were all eligible participants that met the pre-specified entry criteria enrolled?"), 5 (12 out of 45 studies; "Was the sample size sufficiently large to provide confidence in the findings?"), and 8 (12 out of 45 studies; "Were the people assessing the outcomes blinded to the participants' exposures/interventions?”).

\section{Quantitative analysis}

Of the 28 studies involving sedentary/recreationally active subjects and deemed eligible for the qualitative analysis, 21 contributed data to at least one of the planned meta-analyses (Blatchford et al. 1985; Burguera et al. 2000; Carter et al. 2001; Cheneviere et al. 2011; Dasilva et al. 2011; Davis et al. 2000; Devries et al. 2006; 2007; Friedlander et al. 1998, 1999; Henderson et al. 2007; 2008; Keim et al. 1996; Kuo et al. 2005; McKenzie et al. 2000; Mittendorfer et al. 2002; Roepstorff et al. 2006; Steffensen et al. 2002; Tarnopolsky et al. 2007; Venables et al. 2005; White et al. 2003).

Of the 17 studies conducted in athletic populations and deemed eligible for the qualitative analysis, 14 contributed data to at least one of the planned meta-analyses (Abramowicz and Galloway 2005; Goedecke et al. 2000; Horton et al. 2006; Knechtle et al. 2004; Phillips et al. 1993; Powers et al. 1980; Riddell et al. 2003; Roepstorff et al. 2002; Romijn et al. 2000; Steffensen et al. 2002; Tarnopolsky et al. 1990; 1997; Wallis et al. 2006; Zehnder et al. 2005).

Reasons for exclusion from the meta-analyses ranged from 'mixed population' (i.e., enrollment of recreationally active and athletes, without reporting data separately) to presence of sex imbalance (e.g., enrollment of more males than females), as detailed in Fig. 1. Regarding the presence of publication bias in the included studies, for those metaanalyses consisting of at least ten studies, the visual inspection of the funnel plots revealed no asymmetry for all the outcomes considered $\left(\mathrm{VO}_{2}\right.$ peak by body weight and by lean body mass in sedentary subjects; $\mathrm{VO}_{2}$ peak by lean body mass in athletes; carbohydrate raw oxidation in athletes; RER in sedentary subjects; RER in athletes).

Meta-analytic aggregation for sex-based data in sedentary and athletic populations was completed for the following outcomes:
RER Figure 2 show RER results for the comparison between men and women during moderate aerobic exercise in sedentary (12 unique studies, 13 trials, 256 subjects) and athletic (13 unique studies, 14 trials, 251 subjects) populations, respectively. RER was found significantly higher in sedentary men than women (MD: +0.03 ; 95\% CI 0.02-0.04; $p<0.00001$ ), at a moderate to large effect size (SMD: 0.69; 95\% CI 0.42-0.97). Similarly, male athletes displayed a significantly higher RER than women (MD: $+0.02 ; 95 \%$ CI $0.01-0.04 ; p<0.0001)$, at a moderate effect size (SMD: 0.57 ; $95 \%$ CI $0.30-0.83$ ).

Carbohydrate oxidation Percent data pooling from six unique studies (7 trials, 121 subjects) revealed that sedentary men oxidize carbohydrates to a significantly greater extent than their female counterparts, at a moderate effect size (SMD: 0.53 ; 95\% CI 0.15-0.90; $p=0.006$; Fig. 3a). Similarly, the meta-analysis carried out by aggregating raw data from nine unique studies on athletes (10 trials, 156 subjects) showed that male athletes oxidize larger carbohydrates amount than female athletes, at a very large effect size (SMD: 1.24 ; 95\% CI 0.79-1.69; $p<0.00001$; Fig. 3b).

No meta-analyses could be performed for muscle glycogen utilization, as less than three studies shared the same outcome (percent contribution of muscle glycogen to total carbohydrate oxidation; muscle glycogen depletion following exercise; post-exercise muscle glycogen concentration).

Fat oxidation Percent data pooling from eight unique studies (9 trials, 148 subjects) revealed that sedentary men oxidize fat sources to a significantly smaller extent than women, at a large effect size (SMD: $-0.77 ; 95 \%$ CI $-1.18-0.37 ; p=0.0002$; Fig. 4a). On the contrary, data pooling from nine unique studies conducted in athletic populations (10 trials, 154 subjects) showed no difference between male and female athletes in the pattern of fat oxidation. Due to excessive heterogeneity among the studies $\left(\mathrm{I}_{2}=65 \%\right)$ brought by the study by Tarnopolsky et al. (1990), a leave-one-out approach was performed by deleting this study (SMD: 0.06; 95\% CI $-0.37,0.50 ; p=0.77$; Fig. 4b).

Protein oxidation Data on protein oxidation could not be pooled as the two available studies (Horton et al. 1998; Lamont et al. 2001a) enrolled mixed samples including both sedentary and athletic subjects. With specific regard to athletic populations, aggregated data (percent oxidation) from two studies (Horton et al. 2006; Phillips et al. 1993, data not shown) showed a non-significant trend for larger protein oxidation in men than women (SMD: $0.65 ; 95 \%$ CI -0.06 , $1.36 ; p=0.07 ; 33$ subjects).

$\mathrm{VO}_{2}$ peak As expected, maximum oxygen consumption was found significantly higher in sedentary men than women, both when data were normalized to body weight (17 studies, 628 subjects; SMD: 1.18; 95\% CI 0.81, 1.55; $p<0.00001$; $\mathrm{I}^{2}=66 \%$, irreconcilable; Fig. $5 \mathrm{a}$ ) or to lean body mass (16 studies, 595 subjects; SMD: 0.44; 95\% CI 0.12, 0.77; 


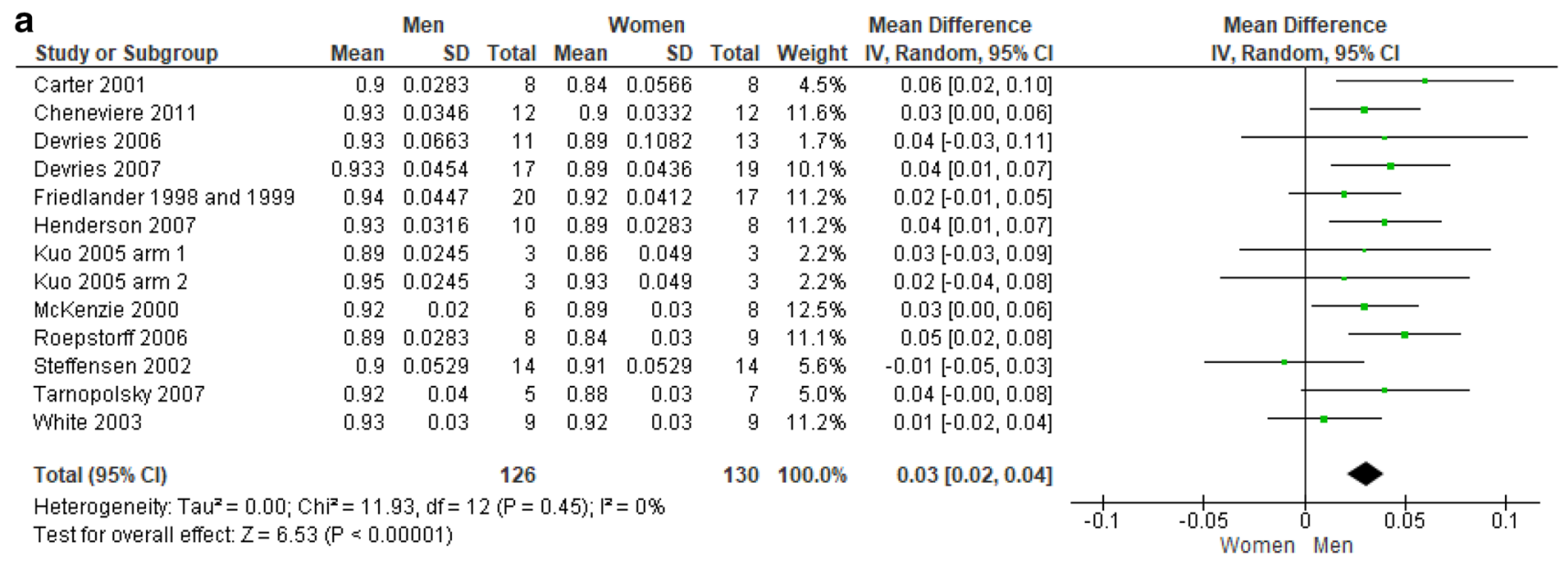

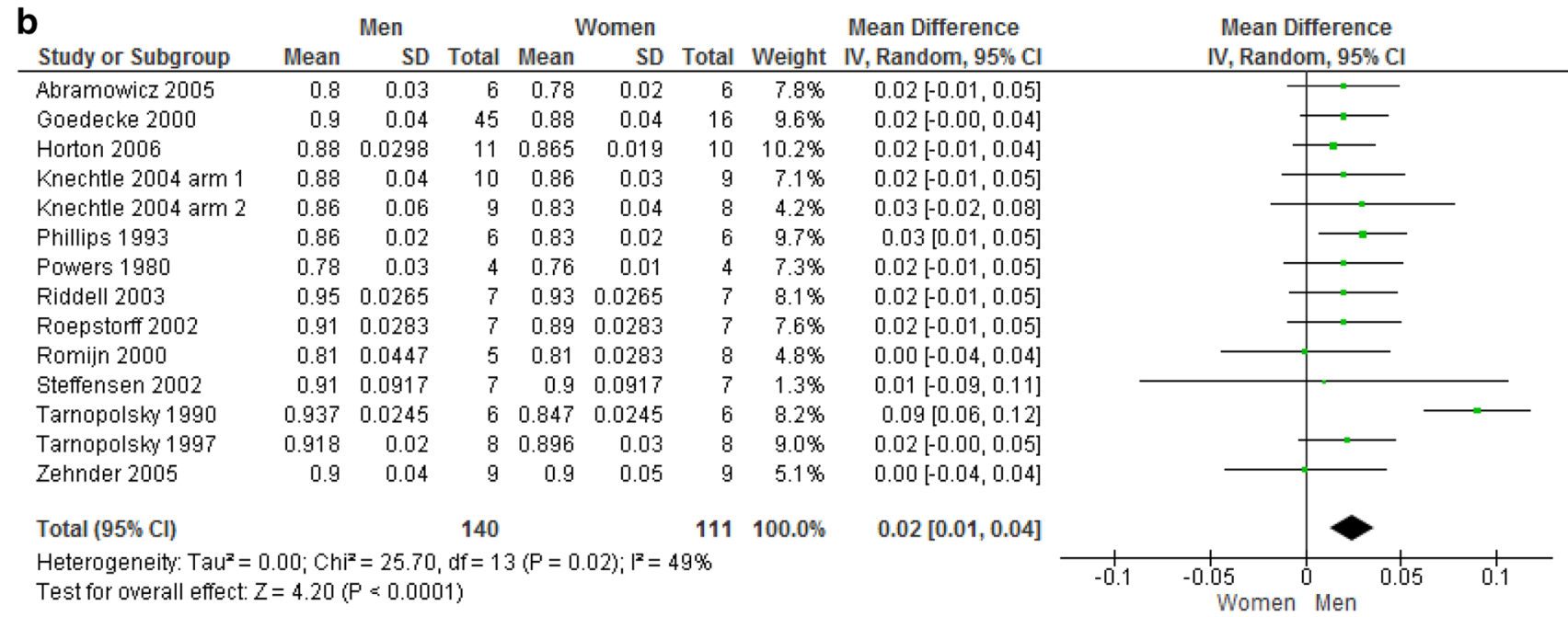

Fig. 2 a Respiratory exchange ratio in sedentary subjects. b Respiratory exchange ratio in athletes

$p=0.008)$. Due to excessive heterogeneity $\left(\mathrm{I}_{2}=61 \%\right)$ among the studies where $\mathrm{VO}_{2}$ was normalized by lean body mass, a leave-one-out approach was performed by deleting the study by Steffensen et al. (2002) and correcting the pooled estimate (15 studies, 567 subjects; SMD: 0.54; 95\% CI 0.24, 0.84; $p=0.0004$; Fig. 5b).

While significantly higher $\mathrm{VO}_{2}$ peak in men was detected also in athletes with data normalized to body weight with a moderate quality of the evidence ( 8 studies, 186 subjects; SMD: 1.30; 95\% CI 0.96, 1.64; $p<0.00001$; Fig. 6a), no sex difference emerged after pooling data normalized to lean body mass ( 11 studies, 186 subjects; SMD: 0.27; 95\% CI -0.09 , $0.62 ; p=0.14)$.

\section{Suggested mechanisms of sex-based differences in substrate utilization}

The main findings of the thematic analysis are graphically summarized in Fig. 7.

Among the 28 studies involving sedentary/recreationally active subjects, the main suggested mechanisms to explain sex dimorphism in fat utilization were differences in "adrenergic activation" (13 studies), "sex hormones" (10 studies), "body composition", and "muscle fiber distribution" (5 studies). Less suggested mechanisms were: "resting substrate content" (i.e., baseline concentration; 2 studies), "different enzymatic activity" (1 study), "mRNA expression of genes 


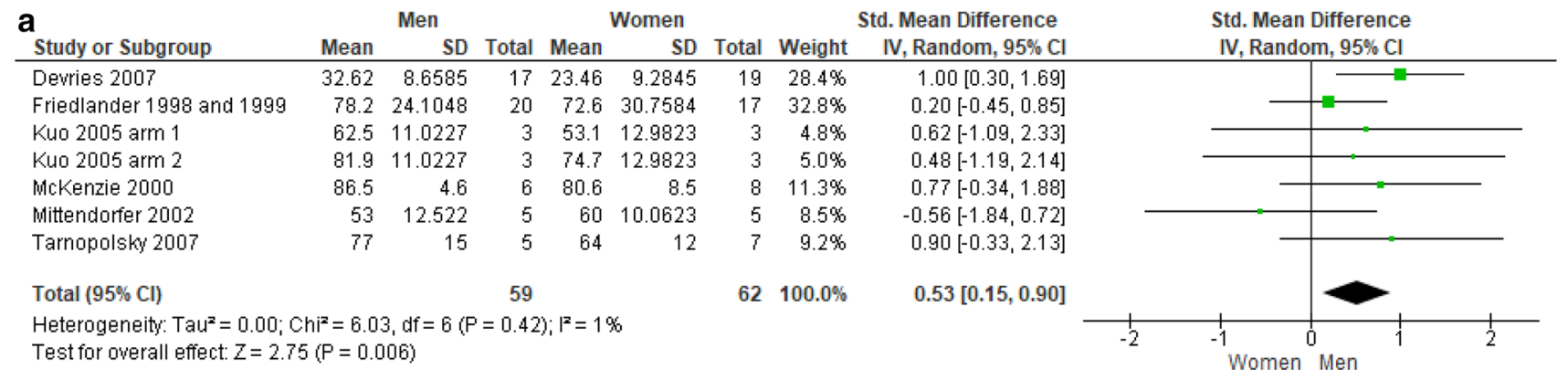

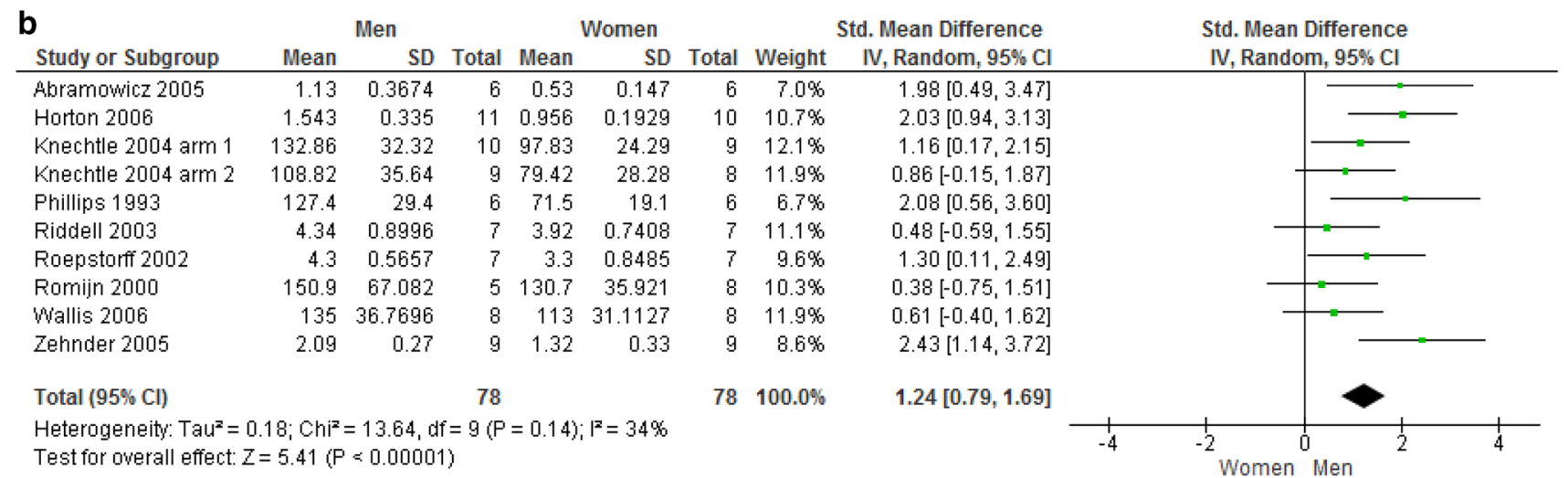

Fig. 3 a Carbohydrate percent oxidation in sedentary subjects. b Carbohydrate raw oxidation in athletes

associated with free fatty acid transport" (e.g., sarcolemmal free fatty acid transport protein and the membrane fatty acid binding protein; 1 study), "sex and adrenergic hormones' interaction" (1 study), "cortisol concentration" (1 study), "higher content of and/or sensitivity to hormone-sensitive lipase (HSL) (1 study), muscle capillarization (1 study).

Regarding carbohydrate utilization, the main suggested mechanisms were differences in "sex hormones" (7 studies), "pattern of glycemic homeostasis maintenance" (i.e., the ability to regain/maintain glycemic homeostasis during exercise and post-exercise recovery; 3 studies), "adrenergic activation", "enzymatic activity" ( 2 studies), "resting substrate content" (i.e., baseline concentration; 1 study), "muscle fiber distribution" (1 study), "receptor availability and affinity" (i.e., the ability of the sex hormonal milieu to modify the concentration of receptors and their ability to bind their specific ligands, modulating substrate utilization; e.g., insulin-binding receptors; 1 study), "mechanism of glucose recycling" (i.e., carbon recycling through gluconeogenesis from lactate, predominantly; 1 study), and "mechanism of hepatic glycogen sparing" (1 study).

Of the 17 studies regarding athletic populations, the most highlighted mechanisms regarding fat utilization in women and men were differences in "adrenergic activation" (4 studies), "muscle fiber distribution", "resting substrate content" (3 studies), "sex hormones" (2 studies); "cortisol concentration" (1 study), and "higher content of and/or sensitivity to HSL (1 study). Sex differences regarding carbohydrate use during exercise were attributed to differences in "sex hormones" (4 studies), "adrenergic activation" (4 studies), "enzymatic activity" (3 studies), "pattern of glycemic homeostasis maintenance" (2 studies), and "muscle fiber distribution" (1 study).

Regarding protein metabolism, the thematic analysis was limited by the paucity of studies available on this topic. The three included studies ( 2 in sedentary subjects; 1 in athletes) converged on "different enzymatic activity" as a candidate mechanism for the observed sex differences in protein metabolism.

\section{Discussion}

The present meta-analysis confirms that both sedentary and athletic males show preferential reliance on carbohydrates to sustain moderate aerobic exercise, while sedentary females rely more on lipids. By contrast, no difference in lipid oxidation rates was observed between male and female athletes, which is a novel finding of the present study.

Regarding the methodological quality of the studies reviewed, the risk for bias in the literature examined was rated as low to moderate. However, failure to clearly define 


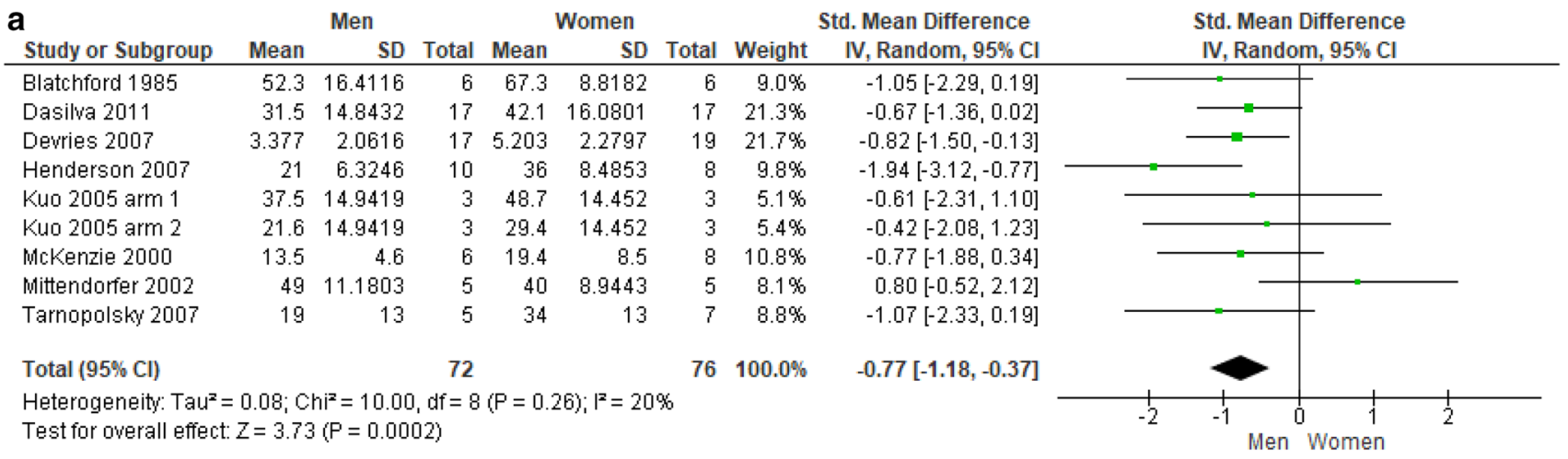

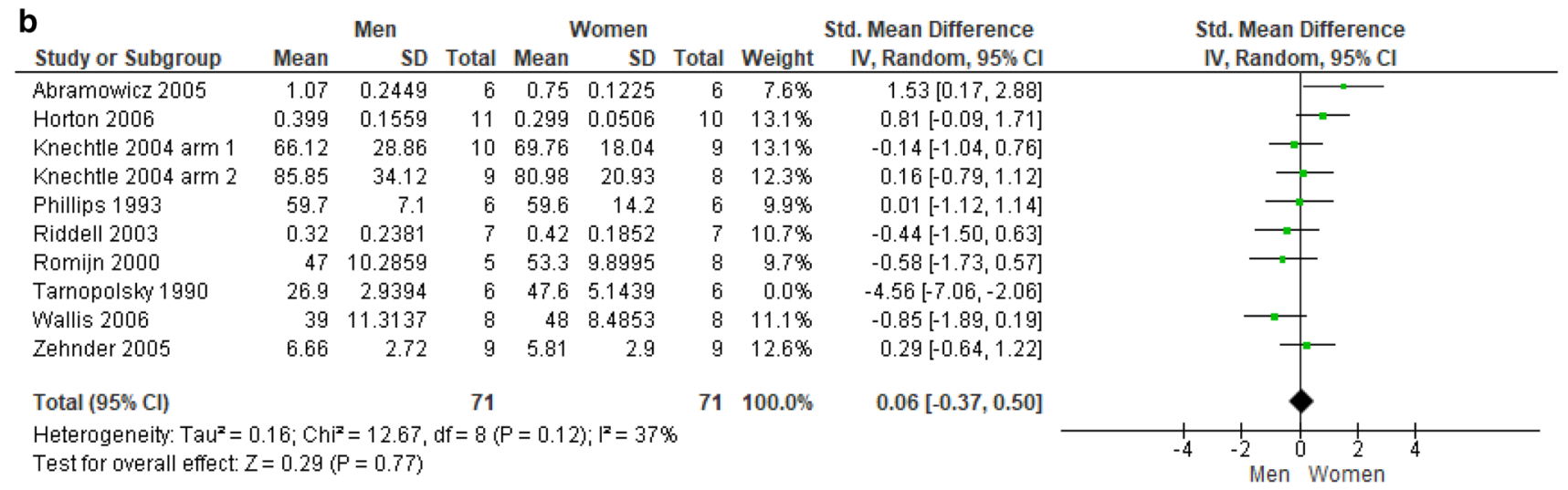

Fig. 4 a Fat percent oxidation in sedentary subjects. b Fat raw oxidation in athletes

inclusion and exclusion criteria for enrollment, limited statistical power, and absence of blinding procedures emerged as the main weaknesses in most of the included studies, thus introducing potential threats to the validity of the results reported by the individual studies.

\section{Sex-based differences in carbohydrate utilization}

Overall, the pooled estimates confirmed the established knowledge that, compared with women, men rely significantly more on whole-body carbohydrate oxidation to sustain moderate-intensity aerobic exercise. This applied both to sedentary and athletic populations, as shown by the higher RER values and the higher percentage of carbohydrates oxidized to sustain the energetic demands. These results are in line with the literature on the topic outlining larger carbohydrate utilization in men by approximately $4-5 \%$ (Tarnopolsky 2000; Devries 2016).

Based on the magnitude of the effect size, reliance on carbohydrates appeared markedly larger among athletes than sedentary/recreationally active subjects. The findings on whole-body carbohydrate utilization are also in line with previous data regarding muscle substrate utilization, fiber types, and enzyme expression/activity. However, these data could not be pooled in our meta-analyses due to excessive methodological heterogeneity or paucity of studies sharing the same outcome measure. Friedlander and colleagues (1998) demonstrated reduced glucose flux and oxidation in women, as assessed by glucose rate of appearance, disappearance, and metabolic clearance. Based on this and other experimental evidence, women are generally reported to utilize 25-50\% less muscle glycogen than matched men during moderate exercise (Tarnopolsky et al. 1990; EsbjörnssonLiljedahl et al. 1999; Devries et al. 2006; Carter et al. 2001).

\section{Sex-based differences in lipid utilization}

Interestingly, the common belief that women tend to rely on lipid sources during moderate aerobic exercise was confirmed in sedentary, but not in athletic populations. An athlete, by definition, is a person who has undertaken training or exercises to become proficient in physical activities such as competitive sports. Athletes are generally considered very fit compared with the general population of same sex and age group (Araújo and Scharhag 2016). The lack of difference between male and female athletes in lipid oxidation may be explained by the increased ability of male athletes to oxidize lipid sources per minute (maximal lipidic power) 


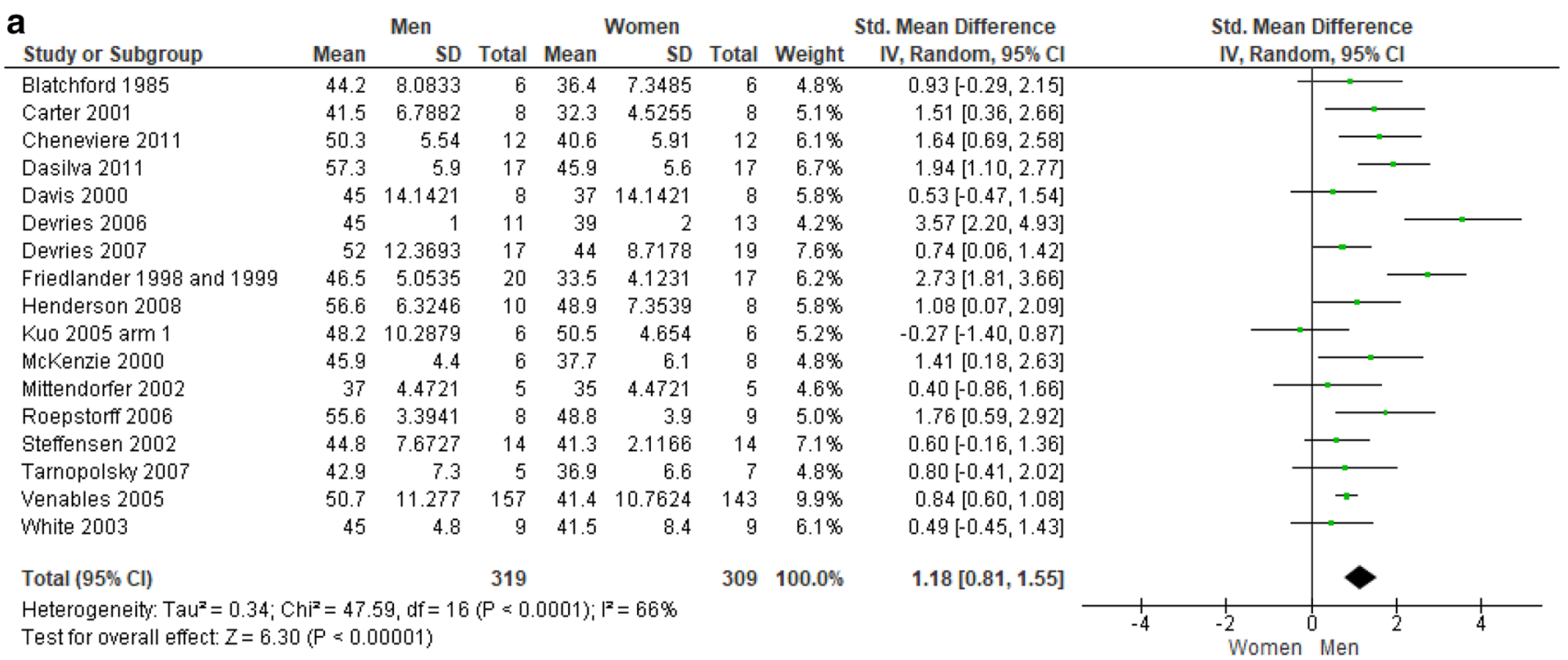

\begin{tabular}{|c|c|c|c|c|c|c|c|}
\hline \multirow{2}{*}{ Study or Subgroup } & \multicolumn{3}{|c|}{ Men } & \multicolumn{3}{|c|}{ Women } & \multirow[b]{2}{*}{ Weight } \\
\hline & Mean & SD & Total & Mean & SD & Total & \\
\hline Blatchford 1985 & 56.1 & 8.8182 & 6 & 52 & 10.5328 & 6 & $4.8 \%$ \\
\hline Burguera 2000 & 56 & 7.3485 & 6 & 51 & 2.4495 & 6 & $4.5 \%$ \\
\hline Carter 2001 & 49.5 & 5.9397 & 8 & 45.7 & 3.3941 & 8 & $5.6 \%$ \\
\hline Cheneviere 2011 & 58.5 & 5.54 & 12 & 55.3 & 6.63 & 12 & $7.4 \%$ \\
\hline Dasilva 2011 & 70.5 & 6.8 & 17 & 58 & 5.9 & 17 & $7.3 \%$ \\
\hline Devries 2006 & 56 & 1 & 11 & 53 & 3 & 13 & $6.7 \%$ \\
\hline Devries 2007 & 61 & 12.3693 & 17 & 58 & 13.0767 & 19 & $9.1 \%$ \\
\hline Henderson 2008 & 63 & 6.957 & 10 & 62.7 & 9.051 & 8 & $6.4 \%$ \\
\hline Keim 1996 & 60.93 & 14.3884 & 10 & 60.54 & 13.9456 & 10 & $6.8 \%$ \\
\hline WhcKenzie 2000 & 54.9 & 3.1 & 6 & 53.4 & 6.4 & 8 & $5.4 \%$ \\
\hline Wittendorfer 2002 & 49 & 4.4721 & 5 & 47 & 2.2361 & 5 & $4.2 \%$ \\
\hline Roepstorff 2006 & 63.4 & 2.2627 & 8 & 65 & 5.1 & 9 & $6.1 \%$ \\
\hline Steffensen 2002 & 53.2 & 6.0852 & 14 & 57 & 5.2915 & 14 & $0.0 \%$ \\
\hline Tamopolsty 2007 & 51.7 & 4.3 & 5 & 49.5 & 6.5 & 7 & $4.8 \%$ \\
\hline Venables 2005 & 62.3 & 10.024 & 157 & 53.5 & 9.5666 & 143 & $14.6 \%$ \\
\hline White 2003 & 51.6 & 4.5 & 9 & 51.9 & 10.2 & 9 & $6.4 \%$ \\
\hline Total $(95 \% \mathrm{Cl})$ & & & 287 & & & 280 & $100.0 \%$ \\
\hline
\end{tabular}

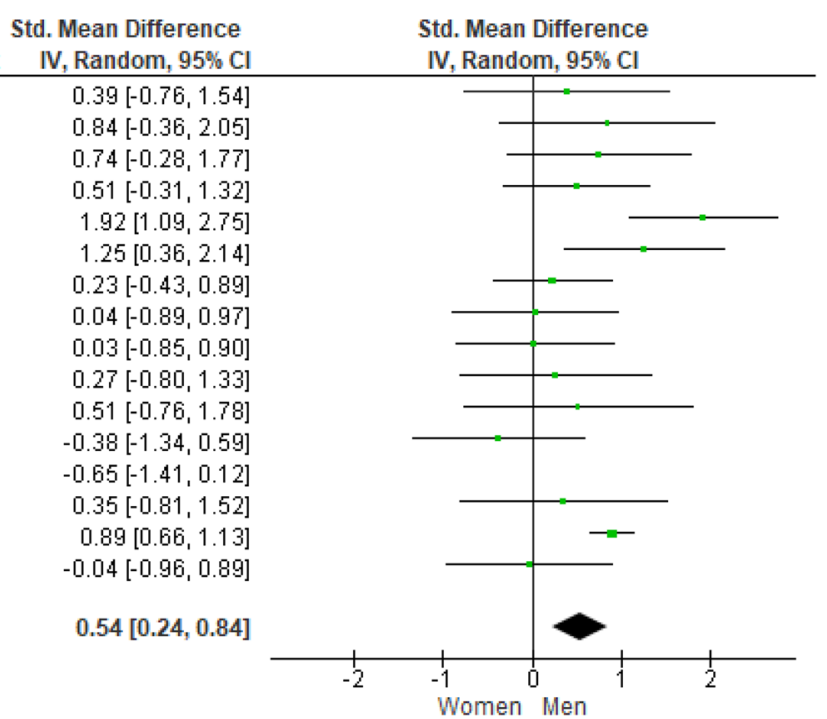

Fig. 5 a Peak oxygen uptake in $\mathrm{ml} / \mathrm{min} / \mathrm{kg}$ in sedentary subjects. b Peak oxygen uptake in $\mathrm{ml} / \mathrm{min} / \mathrm{kg}$ normalized by lean body mass in sedentary subjects

(Gonzalés-Haro et al. 2007). This adaptation might be due to their history of endurance training, compared with sedentary men, who preferentially oxidize carbohydrates.

While this finding has potential implications for training purposes, as male and female athletes exhibit similar fat oxidation rates, it is in discontinuity with a considerable body of literature that reported significantly larger reliance on lipid sources in women than men. Both experimental (Friedlander et al. 1998; Horton et al. 1998; Devries et al. 2007; Henderson et al. 2007; Tarnopolsky et al. 1990; 2007) and knowledge-synthesis works (Tarnopolsky 2000; Devries 2016) demonstrated a significantly lower RER in women, indicating higher whole-body fat oxidation. While the finding on RER was confirmed by our meta-analyses both in sedentary and athletic populations, it disagrees with previous studies that assessed regional substrate utilization, such as IMCL utilization and plasma FFA during endurance exercise. Indeed, both the systemic and leg FFA lipolytic response to aerobic exercise were not different between recreationally active men and women, as stated by Burguera and colleagues (2000). Likewise, FFA utilization was confirmed independent of sex also in athletes, after considering lean body mass differences (Romijn et al. 2000), in line with the findings of the present meta-analysis.

Data collected to examine the effect of sex on IMCL utilization patterns during moderate aerobic exercise are perhaps even more inconclusive. Some works failed to detect differences (White et al. 2003; Devries et al. 2007) 


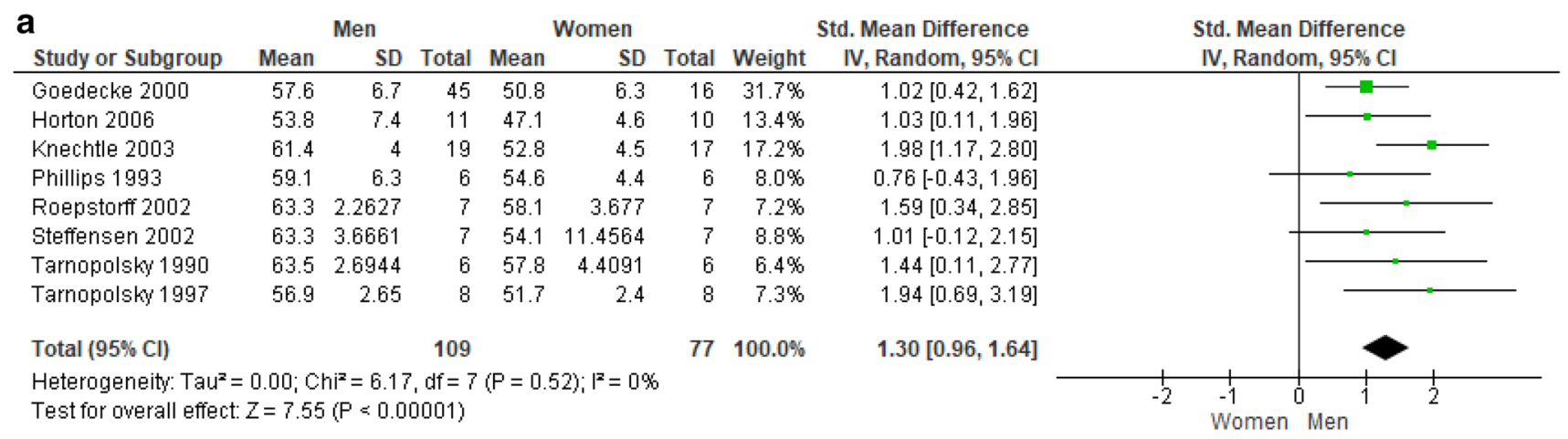

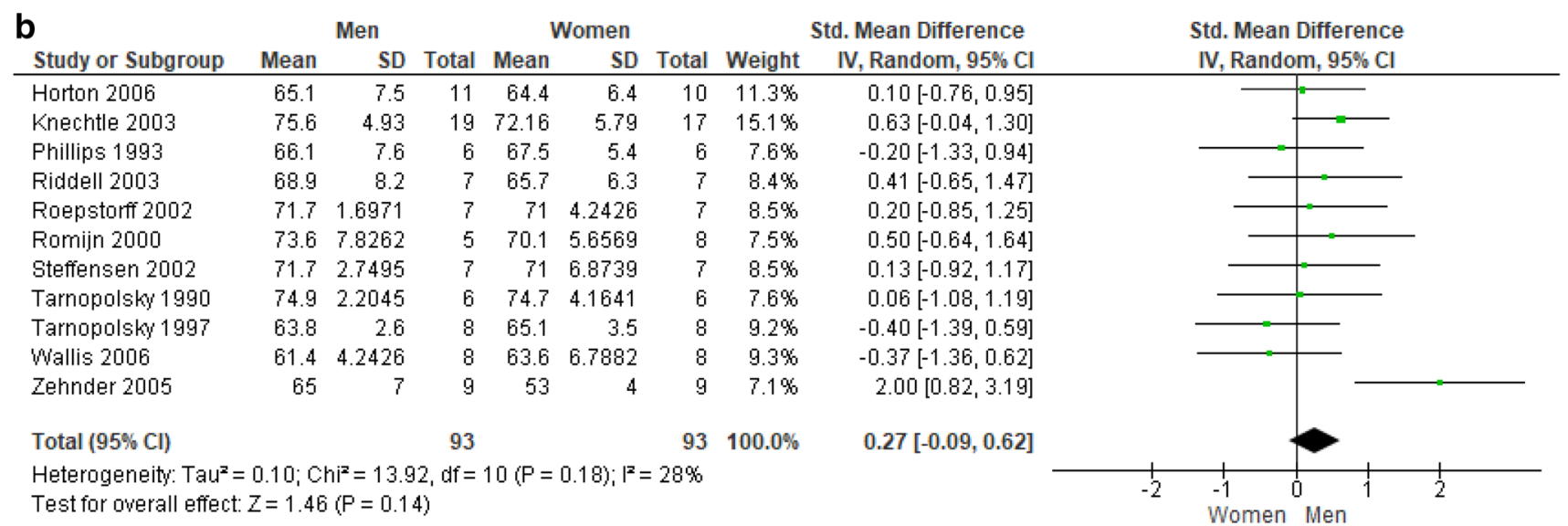

Fig. 6 a Peak oxygen uptake in $\mathrm{ml} / \mathrm{min} / \mathrm{kg}$ in athletic subjects. b Peak oxygen uptake in $\mathrm{ml} / \mathrm{min} / \mathrm{kg}$ normalized by lean body mass in athletic subjects

or found larger (Roepstorff et al. 2002, 2006; Steffensen et al. 2002) or smaller (Zehnder et al. 2005) IMCL utilization in women than men. It has been suggested that methodological inconsistencies and training status differences, between participants within a trial, might contribute to these observed discrepancies (Devries 2016). Possibly for the same reasons, we could not complete a meta-analytical aggregation for FFA and IMCL data, thus preventing to quantify the magnitude of the differences reported in each individual study over a larger pooled sample.

Due to the paucity of sex-comparative studies on protein oxidation patterns during moderate aerobic exercise, no reliable and adequately powered meta-analyses could be performed. Therefore, previous findings from small-sized studies reporting lower oxidation of leucine (Phillips et al. 1993; McKenzie et al. 2000; Lamont et al. 2001a) and greater non-oxidative leucine disposal in women during endurance exercise (Lamont et al. 2001a) could not be confirmed.

\section{Main physiological mechanisms underpinning sex-based differences in substrate utilization}

"Adrenergic activation" emerged as the most cited mechanism responsible for the larger reliance on lipid sources in both sedentary/recreationally active and athletic women. It was also frequently mentioned to partly explain the observed differences in carbohydrate utilization (ranked 3rd in sedentary/recreational, and $2 \mathrm{nd}$ in athletic populations). Tarnopolsky and colleagues (1990) suggested that, while exerciseinduced changes in plasma growth hormone or glucagon concentrations could not explain the greater lipid utilization observed in women, the lower insulin and higher epinephrine concentrations seen in men could partially explain the greater glycogenolysis and glycogen utilization in this group.

Catecholamines are well known to stimulate hepatic glucose production through both increased glycogenolysis and gluconeogenesis. Activation of $\alpha$-adrenoceptors by norepinephrine prompts an increase in blood glucose levels by reducing insulin secretion and glycogenolysis, whereas 


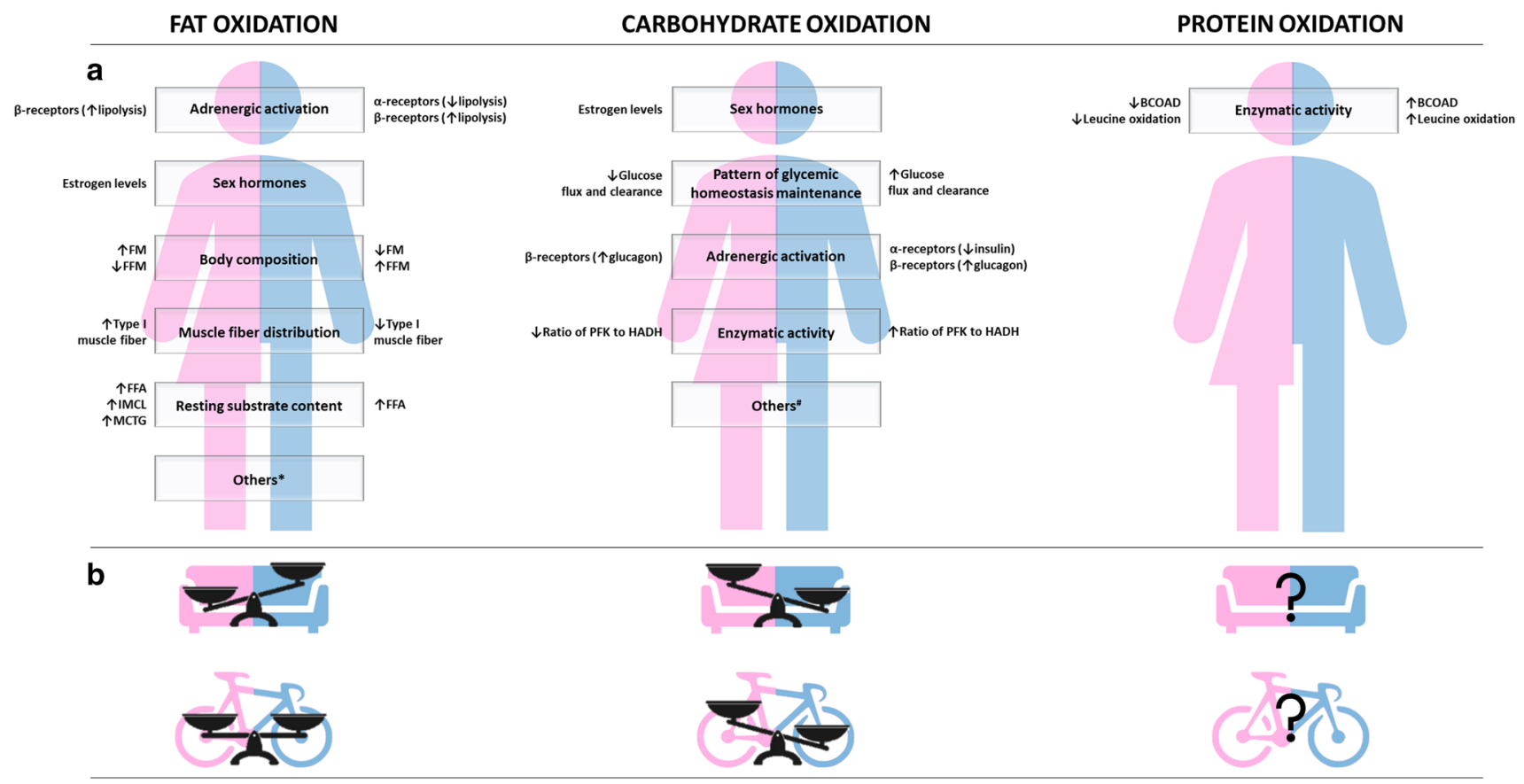

Fig. 7 Graphical overview of the thematic analysis and graphical summary of the meta-analysis results. (A) The thematic analysis highlighted the most cited physiological contributors (boxes) to sex dimorphism in relation to fat, carbohydrate, and protein oxidation, during aerobic moderate-intensity exercise. Associated biological mechanisms that differ between women and men are specified on the left and on the right, respectively. (B) The meta-analysis confirmed sex-based differences in substrate utilization during aerobic moderate-intensity exercise. Sedentary women rely more on fat sources than sedentary men, although this was not confirmed in athletes. Men display greater reliance on carbohydrates than women, as observed both in sedentary (couch) and athletic (bike) populations. Paucity

activation of $\beta$-adrenoceptors contributes to the rise of blood glucose levels by increasing glucagon and adrenocorticotropic hormone secretion (Chu et al. 1996; Horton et al. 2006).

Nevertheless, hormones' biological activity depends not only on circulating concentrations, but also on receptor availability and sensitivity within the individuals. Women may be more sensitive to the lipolytic effects of catecholamines, whereas men may be more sensitive to the hormone's glycolytic effects (Tarnopolsky et al. 1990). From a physiological standpoint, lipolysis in subcutaneous adipose tissue is mainly regulated by adrenergic mechanisms. As introduced earlier, in men, moderate exercise activates $\beta 1$-(lipolysis stimulating) as well as $\alpha 2$-(lipolysis-inhibiting) adrenoceptors, whereas in women only $\beta 1$-receptors are activated, thus supporting their favored kinetic profile of lipid mobilization (Boschmann et al. 2002).

Sex hormones, specifically ovarian hormones, were acknowledged as key contributors to the sex-based differences observed in substrate utilization (ranked 2nd for lipid of studies on protein oxidation prevented meta-analytic aggregation, requiring further research. Others*: enzymatic activity; gene expression; sex and adrenergic hormones' interaction; cortisol; hormonesensitive lipase; muscle capillarization; mRNA expression of genes; receptor availability/affinity. others": resting substrate content; muscle fiber distribution: receptor availability/affinity; glucose recycling; hepatic glycogen sparing; muscle distribution. Abbreviations: FM: fat mass; FFM: free-fat mass; FFA: free fatty acids; IMCL: intramyocellular lipid; MCTG: myocellular triacylglycerol; PFK: phosphofructokinase; HADH: 3-hydroxacyl-CoA dehydrogenase; BCOAD: branched-chain 2-oxoacid dehydrogenase

utilization, in sedentary/recreational populations; 1 st for carbohydrate, both in sedentary/recreational and athletic populations). In women, estrogen directly reduces carbohydrate utilization due to a marked hepatic glycogen sparing effect and insulin-mediated storage, thus indirectly shifting metabolism toward lipids, mainly via FFA mobilization and oxidation (Friedlander 1998; Horton et al. 1998; Carter et al. 2001). Additionally, evidence indicates that women, in comparison to men, have more and larger adipocytes in the gluteal region, which display greater sensitivity to lipolytic agents, such as sex hormones and catecholamines, compared to adipose cells in other sites. Consequently, women display more pronounced regional differences in the hormonal regulation of lipolysis than men during exercise (Blatchford et al. 1985; Arner et al. 1990).

Although relatively minor, compared to sex hormones and adrenergic mechanisms, "muscle fiber distribution" was another factor that emerged from our thematic analysis. Several included studies partly explained sex dimorphism in lipid oxidation based on the established evidence that 
women have a higher percentage of type I highly oxidative low glycolytic fibers, whereas men display a significantly higher proportion of type II highly glycolytic low oxidative fibers (Steffensen et al. 2002). The typical fiber distribution in women is type I > type IIA > type IIX compared to men with type IIA $>$ type I $>$ type IIX (Staron et al. 2000). This evidence would explain why women can oxidize more fat in their muscles, exhibiting reduced muscle fatigability during moderate exercise, while men's metabolism is shifted toward glycolysis to obtain energy (Tarnopolsky et al. 1990; Zierath and Hawley 2004).

Finally, resting substrate content emerged as another mechanism mediating the sex-based differences in substrate utilization. It has been claimed that the higher lipolysis rates in women may partly relate to the larger availability of lipid substrates to support endurance exercise. While women have greater storages of IMCL (Roepstorff et al. 2002; Devries et al. 2007), their greater capacity to use this substrate is still debated, as some studies failed to detect sex differences (White et al. 2003; Devries et al. 2007). However, women have a greater percentage of IMCL in direct contact with mitochondria after a bout of endurance exercise compared with men, which suggests that they may have a greater capacity to use IMCL (Devries 2016) and, thus, a metabolic advantage for endurance when exercising at matched relative intensities (Boschmann et al. 2002; Tarnopolsky et al. 2007).

Women were found to rely more on fat as energy source, thereby using less carbohydrate, amino acid, and protein compared with male exercisers (Phillips et al. 1993; Lamont et al. 2001a). The precise mechanism for the sex difference in protein utilization is still debated. However, the percent activation of hepatic branched-chain 2-oxoacid dehydrogenase appears higher in men, in line with the findings by McKenzie and colleagues (2000). Given the paucity of data on the protein kinetics of men and women during moderate endurance exercise, further sex-comparative studies on protein metabolism are needed.

\section{Study limitations}

A number of potential limitations to the validity of the pooled estimates, outlined in the present review, should be acknowledged. First, the frequent report of mixed samples (sedentary and recreationally active individuals) in most of the studies that did not focus on athletes. Relatedly, all the studies included in this meta-analysis enrolled young adults (aged 18-35 years), thus making our results not generalizable to all age groups. Second, $10 \%$ of the pertinent studies had to be excluded from the analysis, as they enrolled mainly men as participants. This confirms the marked sex bias affecting the research on strategies intended to improve exercise performance and/or health (Devries 2016; Cugusi et al. 2019). Investigators tend to exclude female participants due to the potential influence of fluctuating ovarian hormones throughout the menstrual cycle and its impact on the outcomes of interest. Indeed, when female participants are included in the studies, a poor consideration and characterization of the ovarian hormonal status, menstrual cycle phases, and use of oral contraceptives can be observed, leading to lack of information and inherent mixed female population (Elliott-Sale et al. 2021). Such heterogeneity and lack of reporting may be a potentially limiting factor for the validity of the pooled estimates here obtained. Third, neither diet assessment nor control (prior to exercise testing) were consistently reported by the studies, introducing a certain degree of methodological heterogeneity that may have limited the accuracy of some of the estimates here outlined. Fourth, another element that potentially limits the strength of the findings in athletic populations relates to the exclusion of studies that involved nutritional interventions or supplementation. For those works that planned such interventions, we only considered data from the study arm (if any) where participants were given plain water. Finally, while the range between 45 and $65 \%$ of peak aerobic capacity is well accepted to resemble moderate-intensity aerobic exercise in untrained individuals, this may not apply to endurancetrained subjects who may display high anaerobic threshold, requiring a higher intensity (i.e., $70-75 \%$ of $\mathrm{VO}_{2}$ peak) to match "moderate" aerobic exercise.

\section{Conclusions and future directions}

Meta-analytical aggregations confirmed the occurrence of sex-based differences in fuel utilization during moderate aerobic exercise. Men display higher RER and, accordingly, greater reliance on carbohydrates, whereas sedentary women rely more on fat sources. However, the latter finding was not confirmed in athletes, which is a novel aspect of the present study that requires future tailored investigations. Overall, carbohydrate and lipid kinetics of utilization, during endurance exercise, have been extensively investigated. As emerged, this does not apply to protein metabolism, for evident paucity of data, requiring further research.

The analysis of the main suggested physiological mechanisms related to sex-based difference in substrate utilization during exercise has highlighted the need for mechanistically driven research. Future investigation should not only focus on whole-body substrate utilization patterns, but also include organ-, histological- and cellular-level outcomes, the latter being frequently neglected for lipid and protein metabolism both in sedentary and athletic populations. Moreover, the nutritional status (e.g., body composition, food intake, energy expenditure, pre-testing diet) should be taken into proper consideration since the planning stage of the study, as it can affect substrate metabolism and resting substrate storage. 
To reduce the overall heterogeneity of the existing body of literature on the topic and to improve our understanding of the sex-based differences in substrate utilization, future studies should: (a) consider the diversity and complexities associated with female endocrinology across the lifespan (e.g., menstrual cycle, hormonal contraceptive use, pregnancy, menopause), (b) effectively adapt experimental designs to incorporate female-specific considerations, and (c) clearly characterize female populations included in the study, using the appropriate nomenclature.

Therefore, we recommend that upcoming studies involving women in sport and exercise science adhere to the most recent working guide for standards of practice on the topic (Elliott-Sale et al. 2021). Moreover, to assess the menstrual cycle status and phases, we recommend following the methodological guidance by Janse de Jonge et al. (2019).

Overall, these implementations will likely provide useful information for tailored nutritional and exercise interventions for men and women, addressed toward both the maintenance of good health status and performance improvement.

\section{Acknowledgements None.}

Author contribution $\mathrm{AC}, \mathrm{FD}$, and $\mathrm{AM}$ : conceived and designed the research. AC, LV, GM, and LC: searched the literature and extracted data. LC, MC, and AM: analyzed data. AC, MC, FD, and AM: drafted the manuscript. All authors read and approved the final version of the manuscript.

Funding Open access funding provided by Università degli Studi di Sassari within the CRUI-CARE Agreement. None.

\section{Declarations}

Conflict of interest We certify that no party having a direct interest in the results of the research supporting this article has or will confer a benefit on us or on any organization with which we are associated. All authors declare that they have no conflict of interest.

Ethical approval All procedures performed in studies involving human participants were in accordance with the ethical standards of the institutional and/or national research committee and with the 1964 Helsinki Declaration and its later amendments or comparable ethical standards.

Open Access This article is licensed under a Creative Commons Attribution 4.0 International License, which permits use, sharing, adaptation, distribution and reproduction in any medium or format, as long as you give appropriate credit to the original author(s) and the source, provide a link to the Creative Commons licence, and indicate if changes were made. The images or other third party material in this article are included in the article's Creative Commons licence, unless indicated otherwise in a credit line to the material. If material is not included in the article's Creative Commons licence and your intended use is not permitted by statutory regulation or exceeds the permitted use, you will need to obtain permission directly from the copyright holder. To view a copy of this licence, visit http://creativecommons.org/licenses/by/4.0/.

\section{References}

Abramowicz WN, Galloway SD (2005) Effects of acute versus chronic L-carnitine L-tartrate supplementation on metabolic responses to steady state exercise in males and females. Int J Sport Nutr Exerc Metab 15:386-400

Araújo CG, Scharhag J (2016) Athlete: a working definition for medical and health sciences research. Scand J Med Sci Sports 26:4-7

Arner P, Kriegholm E, Engfeldt P, Bolinder J (1990) Adrenergic regulation of lipolysis in situ at rest and during exercise. J Clin Invest 85:893-898

Bergström J, Hultman E (1966) Muscle glycogen synthesis after exercise: an enhancing factor localized to the muscle cells in man. Nature 210:309-310

Blatchford FK, Knowlton RG, Schneider DA (1985) Plasma FFA responses to prolonged walking in untrained men and women. Eur J Appl Physiol Occup Physiol 53:343-347

Borenstein M, Hedges LV, Higgins JP, Rothstein HR (2010) A basic introduction to fixed-effect and random-effects models for metaanalysis. Res Synth Methods 1:97-111

Boschmann M, Rosenbaum M, Leibel RL, Segal KR (2002) Metabolic and hemodynamic responses to exercise in subcutaneous adipose tissue and skeletal muscle. Int J Sports Med 23:537-543

Burguera B, Proctor D, Dietz N, Guo Z, Joyner M, Jensen MD (2000) Leg free fatty acid kinetics during exercise in men and women. Am J Physiol Endocrinol Metab 278:E113-117

Carter SL, Rennie C, Tarnopolsky MA (2001) Substrate utilization during endurance exercise in men and women after endurance training. Am J Physiol Endocrinol Metab 280:E898-907

Chenevière X, Borrani F, Sangsue D, Gojanovic B, Malatesta D (2011) Gender differences in whole-body fat oxidation kinetics during exercise. Appl Physiol Nutr Metab 36:88-95

Chu CA, Sindelar DK, Neal DW, Cherrington AD (1996) Direct effects of catecholamines on hepatic glucose production in conscious dog are due to glycogenolysis. Am J Physiol 271:127-137

Costill DL, Fink WJ, Getchell LH, Ivy JL, Witzmann FA (1979) Lipid metabolism in skeletal muscle of endurance-trained males and females. J Appl Physiol Respir Environ Exerc Physiol 47:787-791

Cugusi L, Mercuro G (2019) A systematic overview to quantify the gender imbalance in cardiovascular rehabilitation trials. Eur J Prev Cardiol 26:776-781

Dasilva SG, Guidetti L, Buzzachera CF et al (2011) Gender-based differences in substrate use during exercise at a self-selected pace. J Strength Cond Res 25:2544-2551

Davis SN, Galassetti P, Wasserman DH, Tate D (2000) Effects of gender on neuroendocrine and metabolic counterregulatory responses to exercise in normal man. J Clin Endocrinol Metab 85:224-230

D'Eon TM, Sharoff C, Chipkin SR, Grow D, Ruby BC, Braun B (2002) Regulation of exercise carbohydrate metabolism by estrogen and progesterone in women. Am J Physiol Endocrinol Metab 283:E1046-1055

Devries MC (2016) Sex-based differences in endurance exercise muscle metabolism: impact on exercise and nutritional strategies to optimize health and performance in women. Exp Physiol 101:243-249

Devries MC, Hamadeh MJ, Phillips SM, Tarnopolsky MA (2006) Menstrual cycle phase and sex influence muscle glycogen utilization and glucose turnover during moderate-intensity endurance exercise. Am J Physiol Regul Integr Comp Physiol 291:R1120-1128

Devries MC, Lowther SA, Glover AW, Hamadeh MJ, Tarnopolsky MA (2007) IMCL area density, but not IMCL utilization, is higher in women during moderate-intensity endurance exercise, compared with men. Am J Physiol Regul Integr Comp Physiol 293:R2336-R2342

Elliott-Sale KJ, Minahan CL, de Jonge XAKJ, Ackerman KE, Sipilä S, Constantini NW, Lebrun CM, Hackney AC (2021) 
Methodological considerations for studies in sport and exercise science with women as participants: a working guide for standards of practice for research on women. Sports Med 51:843-861

Esbjörnsson-Liljedahl M, Sundberg CJ, Norman B, Jansson E (1999) Metabolic response in type I and type II muscle fibers during a 30-s cycle sprint in men and women. J Appl Physiol 87:1326-1332

Fletcher GF, Balady GJ, Amsterdam EA, Chaitman B, Eckel R, Fleg J et al (2001) Exercise standards for testing and training: a statement for healthcare professionals from the American Heart Association. Circulation 104:1694-1740

Friedlander AL, Casazza GA, Horning MA, Huie MJ, Piacentini MF, Trimmer JK, Brooks GA (1998) Training-induced alterations of carbohydrate metabolism in women: women respond differently from men. J Appl Physiol 85:1175-1186

Friedlander AL, Casazza GA, Horning MA, Usaj A, Brooks GA (1999) Endurance training increases fatty acid turnover, but not fat oxidation, in young men. J Appl Physiol 86:2097-2105

Goedecke JH, St Clair Gibson A, Grobler L, Collins M, Noakes TD, Lambert EV (2000) Determinants of the variability in respiratory exchange ratio at rest and during exercise in trained athletes. Am J Physiol Endocrinol Metab 279:1325-1334

González-Haro C, Galilea PA, González-de-Suso JM, Drobnic F, Escanero JF (2007) Maximal lipidic power in high competitive level triathletes and cyclists. Br J Sports Med 41:23-28

Grimes DA, Schulz KF (2002) Bias and causal associations in observational research. Lancet 359:248-252

Hackney AC (1999) Influence of oestrogen on muscle glycogen utilization during exercise. Acta Physiol Scand 167:273-274

Hellström L, Blaak E, Hagström-Toft E (1996) Gender differences in adrenergic regulation of lipid mobilization during exercise. Int $\mathbf{J}$ Sports Med 17:439-447

Henderson GC, Fattor JA, Horning MA et al (2007) Lipolysis and fatty acid metabolism in men and women during the postexercise recovery period. J Physiol 584:963-981

Henderson GC, Fattor JA, Horning MA et al (2008) Glucoregulation is more precise in women than in men during postexercise recovery. Am J Clin Nutr 87:1686-1694

Higgins JP, Thompson SG, Deeks JJ, Altman DG (2003) Measuring inconsistency in meta-analyses. BMJ 327:557-560

Horowitz JF, Klein S (2000) Oxidation of nonplasma fatty acids during exercise is increased in women with abdominal obesity. J Appl Physiol 89:2276-2282

Horton TJ, Grunwald GK, Lavely J, Donahoo WT (2006) Glucose kinetics differ between women and men, during and after exercise. J Appl Physiol 100:1883-1894

Horton TJ, Pagliassotti MJ, Hobbs K, Hill JO (1998) Fuel metabolism in men and women during and after long-duration exercise. J Appl Physiol 85:1823-1832

Hunter SK (2014) Sex differences in human fatigability: mechanisms and insight to physiological responses. Acta Physiol (oxf) 210:768-789

Isacco L, Duché P, Boisseau N (2012) Influence of hormonal status on substrate utilization at rest and during exercise in the female population. Sports Med 42:327-342

Isacco L, Ennequin G, Boisseau N (2020) Effect of Fat Mass Localization on Fat Oxidation During Endurance Exercise in Women. Front Physiol 11:585137

Janse DE, Jonge X, Thompson B, Han A (2019) Methodological Recommendations for Menstrual Cycle Research in Sports and Exercise. Med Sci Sports Exerc 51:2610-2617

Jensen MD, Cryer PE, Johnson CM, Murray MJ (1996) Effects of epinephrine on regional free fatty acid and energy metabolism in men and women. Am J Physiol Endocrinol Metab 270:259-264

Keim NL, Belko AZ, Barbieri TF (1996) Body fat percentage and gender: associations with exercise energy expenditure, substrate utilization, and mechanical work efficiency. Int J Sport Nutr $6: 356-369$

Kiens B, Roepstorff C, Glatz JFC, Bonen A, Schjerling P, Knudsen J et al (2004) Lipid-binding proteins and lipoprotein lipase activity in human skeletal muscle: Influence of physical activity and gender. J Appl Physiol 97:1209-1218

Knechtle B, Müller G, Willmann F, Kotteck K, Eser P, Knecht H (2004) Fat oxidation in men and women endurance athletes in running and cycling. Int J Sports Med 25:38-44

Kuo CC, Fattor JA, Henderson GC, Brooks GA (2005) Lipid oxidation in fit young adults during postexercise recovery. J Appl Physiol 99:349-356

Lamont LS, McCullough AJ, Kalhan SC (2001a) Gender differences in leucine, but not lysine, kinetics. J Appl Physiol 91:357-362

Lamont LS, McCullough AJ, Kalhan SC (2001b) Relationship between leucine oxidation and oxygen consumption during steady-state exercise. Med Sci Sports Exerc 33:237-241

Lamont LS, McCullough AJ, Kalhan SC (2003) Gender differences in the regulation of amino acid metabolism. J Appl Physiol 95:1259-1265

Liberati A, Altman DG, Tetzlaff J, Mulrow C, Gøtzsche PC, Ioannidis JP, Clarke M, Devereaux PJ, Kleijnen J, Moher D (2009) The PRISMA statement for reporting systematic reviews and metaanalyses of studies that evaluate health care interventions: explanation and elaboration. J Clin Epidemiol 62:e1-34

McKenzie S, Phillips SM, Carter SL, Lowther S, Gibala MJ, Tarnopolsky MA (2000) Endurance exercise training attenuates leucine oxidation and BCOAD activation during exercise in humans. Am J Physiol Endocrinol Metab 278:580-587

Millet L, Barbe P, Lafontan M, Berlan M, Galitzky J (1998) Catecholamine effects on lipolysis and blood flow in human abdominal and femoral adipose tissue. J Appl Physiol 85:181-188

Mittendorfer B, Horowitz JF, Klein S (2002) Effect of gender on lipid kinetics during endurance exercise of moderate intensity in untrained subjects. Am J Physiol Endocrinol Metab 283:E58-E65

Monaco C, Whitfield J, Jain SS, Spriet LL, Bonen A, Holloway GP (2015) Activation of $\mathrm{AMPK} \alpha 2$ is not required for mitochondrial FAT/CD36 accumulation during exercise. PLoS ONE 10:e0126122

Oosthuyse T, Bosch AN (2010) The effect of the menstrual cycle on exercise metabolism: implications for exercise performance in eumenorrhoeic women. Sports Med 40:207-227

Phillips SM, Atkinson SA, Tarnopolsky MA, MacDougall JD (1993) Gender differences in leucine kinetics and nitrogen balance in endurance athletes. J Appl Physiol 75:2134-2141

Powers SK, Riley W, Howley ET (1980) Comparison of fat metabolism between trained men and women during prolonged aerobic work. Res Q Exerc Sport 51:427-431

Riddell MC, Partington SL, Stupka N, Armstrong D, Rennie C, Tarnopolsky MA (2003) Substrate utilization during exercise performed with and without glucose ingestion in female and male endurance trained athletes. Int J Sport Nutr Exerc Metab 13:407-421

Roepstorff C, Steffensen CH, Madsen M, Stallknecht B, Kanstrup IL, Richter EA, Kiens B (2002) Gender differences in substrate utilization during submaximal exercise in endurance-trained subjects. Am J Physiol Endocrinol Metab 282:E435-447

Roepstorff C, Donsmark M, Thiele M, Vistisen B, Stewart G, Vissing K, Schjerling P, Hardie DG, Galbo H, Kiens B (2006) Sex differences in hormone-sensitive lipase expression, activity, and phosphorylation in skeletal muscle at rest and during exercise. Am J Physiol Endocrinol Metab 291:E1106-1114

Romijn JA, Coyle EF, Sidossis LS, Rosenblatt J, Wolfe RR (2000) Substrate metabolism during different exercise intensities in endurance-trained women. J Appl Physiol 88:1707-1714 
Ruby BC, Coggan AR, Zderic TW (2002) Gender differences in glucose kinetics and substrate oxidation during exercise near the lactate threshold. J Appl Physiol 92:1125-1132

Simunovic N, Sprague S, Bhandari M (2009) Methodological issues in systematic reviews and meta-analyses of observational studies in orthopaedic research. J Bone Joint Surg Am 91:87-94

Spriet LL (2014) New insights into the interaction of carbohydrate and fat metabolism during exercise. Sports Med 44:S87-96

Staron RS, Hagerman FC, Hikida RS, Murray TF, Hostler DP, Crill MT, Ragg KE, Toma K (2000) Fiber type composition of the vastus lateralis muscle of young men and women. J Histochem Cytochem 48:623-629

Steffensen CH, Roepstorff C, Madsen M, Kiens B (2002) Myocellular triacylglycerol breakdown in females but not in males during exercise. Am J Physiol Endocrinol Metab 282:E634-642

Sterne JA, Sutton AJ, Ioannidis JP, Terrin N, Jones DR, Lau J, Carpenter J, Rücker G, Harbord RM, Schmid CH, Tetzlaff J, Deeks JJ, Peters J, Macaskill P, Schwarzer G, Duval S, Altman DG, Moher D, Higgins JP (2011) Recommendations for examining and interpreting funnel plot asymmetry in meta-analyses of randomised controlled trials. BMJ 22:343

Tarnopolsky MA (2000) Gender differences in substrate metabolism during endurance exercise. Can J Appl Physiol 25:312-327

Tarnopolsky LJ, MacDougall JD, Atkinson SA, Tarnopolsky MA, Sutton JR (1990) Gender differences in substrate for endurance exercise. J Appl Physiol 68:302-308

Tarnopolsky MA, Bosman M, Macdonald JR, Vandeputte D, Martin J, Roy BD (1997) Postexercise protein-carbohydrate and carbohydrate supplements increase muscle glycogen in men and women. J Appl Physiol 83:1877-1883

Tarnopolsky MA, Rennie CD, Robertshaw HA, Fedak-Tarnopolsky SN, Devries MC, Hamadeh MJ (2007) Influence of endurance exercise training and sex on intramyocellular lipid and mitochondrial ultrastructure, substrate use, and mitochondrial enzyme activity. Am J Physiol Regul Integr Comp Physiol 292:R1271-R1278

Temesi J, Arnal PJ, Rupp T, Féasson L, Cartier R, Gergelé L et al (2015) Are females more resistant to extreme neuromuscular fatigue? Med Sci Sports Exerc 47:1372-1382

Tiller NB, Elliott-Sale KJ, Knechtle B, Wilson PB, Roberts JD, Millet GY (2021) Do sex differences in physiology confer a female advantage in ultra-endurance sport? Sports Med. https://doi.org/ 10.1007/s40279-020-01417-2

Venables MC, Achten J, Jeukendrup AE (2005) Determinants of fat oxidation during exercise in healthy men and women: a crosssectional study. J Appl Physiol 98:160-167

Wallis GA, Dawson R, Achten J, Webber J, Jeukendrup AE (2006) Metabolic response to carbohydrate ingestion during exercise in males and females. Am J Physiol Endocrinol Metab 290E708-E715

White LJ, Ferguson MA, McCoy SC, Kim H (2003) Intramyocellular lipid changes in men and women during aerobic exercise: a (1)H-magnetic resonance spectroscopy study. J Clin Endocrinol Metab 88:5638-5643

Zehnder M, Ith M, Kreis R, Saris W, Boutellier U, Boesch C (2005) Gender-specific usage of intramyocellular lipids and glycogen during exercise. Med Sci Sports Exerc 37:1517-1524

Zierath JR, Hawley JA (2004) Skeletal muscle fiber type: influence on contractile and metabolic properties. PLoS Biol 2:e348

Publisher's Note Springer Nature remains neutral with regard to jurisdictional claims in published maps and institutional affiliations. 